\title{
Synthesis and Antimicrobial Studies of Some 4-(Substituted)-Ethanoylamino-3-Mercapto-5- (4-Substituted) Phenyl-1,2,4-Triazoles
}

\author{
Neeraj Upmanyu ${ }^{1}$, Sanjay Kumar ${ }^{2}$, Kamal Shah ${ }^{3}$ and Pradeep Mishra ${ }^{3}$ \\ ${ }^{1}$ Pharmaceutical Chemistry Division, Department of Pharmaceutical Sciences, Dr. H.S. Gour University, \\ Sagar-470003, India \\ ${ }^{2}$ Defence Research Development and Establishment, Jhansi Road, Gwalior-474001, India \\ ${ }^{3}$ GLA Institute of Pharmaceutical Research, Mathura-281406, India
}

\begin{abstract}
Triazoles and triazoles with different substituent groups are found to possess diverse application in the field of medicine and industry. A series of 4-(substituted) ethanoylamino-3-mercapto-5-(4-substituted) phenyl-1,2,4triazoles were synthesized as novel antimicrobial agents starting from different 4-substituted benzoic acids. The chemical structures of these newly synthesized compounds were elucidated by IR, ${ }^{1} \mathrm{H} N \mathrm{NMR},{ }^{13} \mathrm{C} \mathrm{NMR}, \mathrm{FAB}{ }^{+}$-mass spectral data and elemental analyses. The antimicrobial activity of title compounds were examined against two gram positive bacteria (Staphylococcus aureus, Bacillus subtilis), two gram negative bacteria (Escherichia coli, Pseudomonas aeruginosa) and three fungi (Candida albicans, Aspergillus niger and Fusarium oxysporum) using disc diffusion method. Some of the compounds bearing methoxy group exhibited moderate to good antibacterial and antifungal activities.
\end{abstract}

Key words: 1, 2, 4-Triazoles, zone of inhibition, antibacterial, antifungal.

\section{INTRODUCTION}

Combat against bacterial and fungal infection has resulted in the development of a wide variety of antimicrobial drugs. After years of misuse and overuse of these drugs, microorganisms are becoming resistant resulting in a potent global health problem. It is evident that antimicrobial resistance is associated with an increase in mortality. ${ }^{1}$ Hence, there is still a critical need for new antimicrobial agents to treat life-threatening diseases. In recent years active research has been initiated on heterocyclic chemistry and thus, scientists are engaged to synthesize new compounds related to various chemical groups like indole, 1,2,4-triazole, thiazole, imidazole, azetidinone, etc. 1,2,4-Triazoles have received considerable attention owing to their synthetic and effective biological importance. 1,2,4-Triazole derivatives are well known in medicinal chemistry due to their diverse biological properties like

Correspondence to: Neeraj Upmanyu

Mob. No.: 9425128642

Email: drneerajupmanyu@gmail.com

Dhaka Univ. J. Pharm. Sci. 11(1): 7-18, 2012 (June) antibacterial, antifungal, ${ }^{2-8}$ antitubercular, ${ }^{9,10}$ anticancer, ${ }^{11}$ anti-tumor, ${ }^{12}$ anti-inflammatory ${ }^{13-15}$, anticonvulsant, ${ }^{16-17}$ urease inhibitor, ${ }^{18}$ openers of Caactivated potassium (Maxi-K) channel, ${ }^{19}$ tubulin polymerization inhibitors, ${ }^{20}$ ghrelin receptor antagonist ${ }^{21}$ and antiviral activities. ${ }^{22}$

These findings prompted us to synthesize 4(substituted) ethanoylamino-3-mercapto-5-(4substituted) phenyl-1,2,4-triazoles 6-14a,b having substitution on aromatic ring and different secondary amino groups were attached on ethanoyl moiety and these compounds were evaluated for antimicrobial activity.

\section{MATERIALS AND METHODS}

Thin layer chromatography was used to observe completion of the reaction and purity of the synthesized compounds. Melting points were determined in open glass capillary tubes by using melting point apparatus and were uncorrected. IR spectra in $\mathrm{KBr}$ were recorded on a Nicolet-6700 
FTIR spectrophotometer. The ${ }^{1} \mathrm{H}$ - and ${ }^{13} \mathrm{C}-\mathrm{NMR}$ spectra were recorded on Bruker avance III $400 \mathrm{MHZ}$ spectrophotometer in DMSO- $\mathrm{d}_{6} / \mathrm{CDCl}_{3}$ using TMS as an internal standard (chemical shifts are expressed in $\delta, \mathrm{ppm}$ ), Mass spectra were recorded on Jeol Sx 102/DA-600 mass spectrometer/data system using fast moving bombardment (FAB) technique and nitrogen analysis was recorded using elemental analyzer Elementar Vario EL III Carlo Erba 1108.
The purity of the compounds was confirmed by TLC using Merck silica gel $60 \mathrm{~F}_{254}$ plates using a mixture of ethyl acetate and petroleum ether (1:1) and spots were visualized under UV radiation.

The synthesis of 4-(substituted ethanoyl) amino3-mercapto-5-(4-substituted) phenyl-1,2,4-triazoles (6a-o) were prepared following stepwise procedures as mentioned in Figure 1.

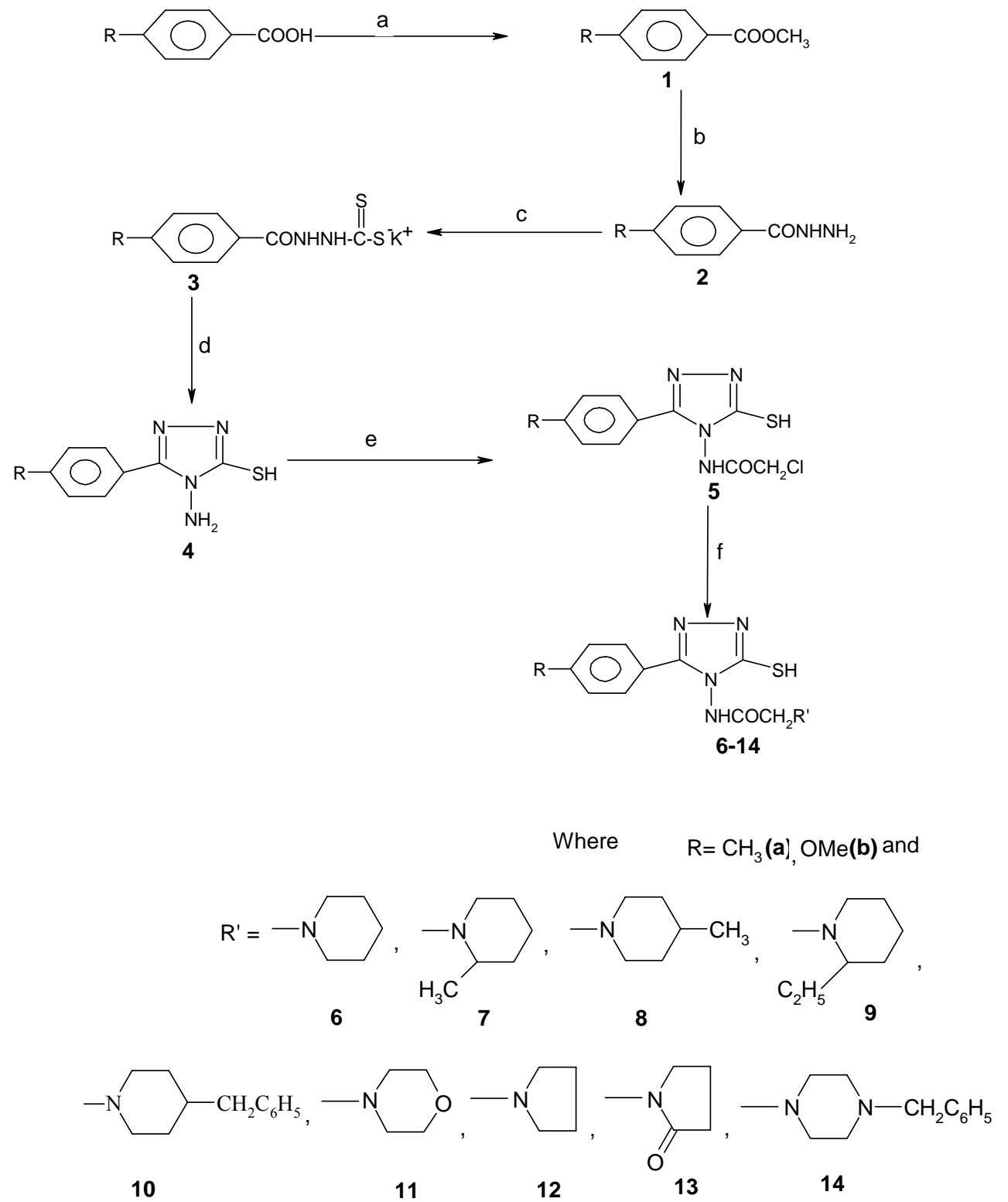

Reagents and conditions : a) $\mathrm{CH}_{3} \mathrm{OH}$ and conc. $\mathrm{H}_{2} \mathrm{SO}_{4}$, b) $\mathrm{NH}_{2} \mathrm{NH}_{2} \mathrm{H}_{2} \mathrm{O}$, c) $\mathrm{CS}_{2}$ and alc. $\mathrm{KOH}$, d) $\mathrm{NH}_{2} \mathrm{NH}_{2} \mathrm{H}_{2} \mathrm{O}$ and $\mathrm{C}_{2} \mathrm{H}_{5} \mathrm{OH}$, e) $\mathrm{ClCH}_{2} \mathrm{COCl}$, f) different secondary amines 
General procedure for methyl esters $(\mathbf{1 a}, \mathbf{b})$. Substituted carboxylic acid $(0.1 \mathrm{~mol})$ was taken in methanol $(100 \mathrm{ml})$ in a round bottom flask and conc. sulphuric acid $(5.7 \mathrm{ml})$ was added to that. The mixture was refluxed for 4-6 hr. Excess of methanol was then distilled off. After cooling the contents were transferred to separating funnel containing $100 \mathrm{ml}$ of distilled water. The synthesized ester was repeatedly extracted several times with carbon tetrachloride (30 $\mathrm{ml})$. The combined organic layer was washed with solution of sodium-bi-carbonate (20\%) to remove any unreacted acid. After washing with distilled water the organic layer was dried over anhydrous $\mathrm{MgSO}_{4}$. Carbon tetrachloride was then distilled off under reduced pressure to get the ester, which was recrystallized from absolute alcohol.

Methyl 4-methylbenzoate (1a). Yield 94\%, mp: 34-36 $6^{\circ} \mathrm{C}$. IR (KBr), v cm${ }^{-1}: 2856,2960\left(\mathrm{CH}_{3}\right), 1286$, 1121 (C-O-C), $1722(\mathrm{C}=\mathrm{O})$.

Methyl 4-methoxybenzoate (1b). Yield 78\%, mp: 46-48 ${ }^{\circ} \mathrm{C}$. IR (KBr), v, cm ${ }^{-1}$ : 2852, $2958\left(\mathrm{CH}_{3}\right)$, 1280,1118 (C-O-C), $1720(\mathrm{C}=\mathrm{O})$.

General procedure for hydrazides (2a,b). Hydrazine hydrate $(99 \%)(5.7 \mathrm{ml}, 0.15 \mathrm{~mol})$ was taken in a flat bottom flask and solution of methyl ester 1a,b $(0.1 \mathrm{~mol})$ in ethanol was added dropwise with gentle stirring. After complete addition, the mixture was transferred into a round bottomed flask and refluxed for 4-6 hr. Ethanol was distilled off under reduced pressure. The precipitate formed was filtered and after drying recrystallized from ethanol.

4-Methyl benzohydrate (2a). Yield 93\%, mp: $110-112^{\circ} \mathrm{C}$. IR $(\mathrm{KBr}), v \mathrm{~cm}^{-1}: 1650(\mathrm{C}=\mathrm{O}), 3050$ (Ar$\mathrm{CH}), 3304(\mathrm{NH}), 3435,3345\left(\mathrm{NH}_{2}\right)$.

4-Methoxy benzohydrate (2b). Yield 92\%, mp: $132-134^{\circ} \mathrm{C}$. IR (KBr), v cm ${ }^{-1}: 1642(\mathrm{C}=\mathrm{O}), 3070$ (Ar$\mathrm{CH}), 3310(\mathrm{NH}), 3428,3350\left(\mathrm{NH}_{2}\right)$.

General procedure for potassium 3-aroyl dithiocarbazate $(\mathbf{3 a}, \mathbf{b})$. A mixture of potassium hydroxide $(0.15 \mathrm{~mol}), 100 \mathrm{ml}$ of absolute ethanol and $0.1 \mathrm{~mol}$ of the aroylhydrazide $\mathbf{2} \mathbf{a}, \mathbf{b}$ were treated with $0.15 \mathrm{~mol}$ of carbon disulfide. This mixture was further diluted with $75 \mathrm{ml}$ of absolute ethanol and stirred for 12-16 hr. The solvent was distilled off under reduced pressure. The salts, prepared as described above, were obtained in nearly quantitative yield and were employed without further purification.

General procedure for 4-amino-3-mercapto-5(4-substituted) phenyl-1,2,4-triazole (4a,b). A suspension of $0.1 \mathrm{~mol}$ of the potassium salt $\mathbf{3 a}, \mathbf{b}$ in absolute alcohol, $0.2 \mathrm{~mol}$ of hydrazine hydrate (99\%) and water $(6 \mathrm{ml})$ was refluxed for 2 to $3 \mathrm{hr}$. The colour of the reaction mixture changed to green with the evolution of hydrogen sulfide gas and a homogenous solution resulted. Cold distilled water $(100 \mathrm{ml})$ was added and the solution was acidified with concentrated hydrochloric acid. The precipitated solid was filtered, washed with $2 \times 30 \mathrm{ml}$ portions of cold water and recrystallized from aqueous ethanol $(50 \%)$.

4-Amino-3-mercapto-5-(4-methylphenyl)-1,2,4triazole (4a). Yield $75 \%$, mp: $210-212^{\circ} \mathrm{C}$. IR (KBr), v $\mathrm{cm}^{-1}: 1350(\mathrm{C}-\mathrm{N}), 1540(\mathrm{C}=\mathrm{N}), 3000(\mathrm{Ar}-\mathrm{CH}), 2550$ $(\mathrm{SH}), 3312(\mathrm{NH}), 3432,3346\left(\mathrm{NH}_{2}\right)$.

4-Amino-3-mercapto-5-(4-methoxyphenyl)-1,2,4triazole (4b). Yield $78 \%$, mp: $201-203^{\circ} \mathrm{C}$. IR (KBr), v, $\mathrm{cm}^{-1}: 1345(\mathrm{C}-\mathrm{N}), 1538(\mathrm{C}=\mathrm{N}), 3050(\mathrm{Ar}-\mathrm{CH})$, $2540(\mathrm{SH}), 3310(\mathrm{NH}), 3438,3352\left(\mathrm{NH}_{2}\right), 1262$, 1092 (C-O-C).

General procedure for 4-(chloro-acyl amino)3-mercapto-5-(4-substituted) phenyl-1,2,4-triazole. (5a,b). Compound 4-amino-3-mercapto-5-(psubstituted) phenyl-1,2,4-triazole $\mathbf{4 a , b}(0.1 \mathrm{~mol})$ was taken in dioxane $(50 \mathrm{ml})$ in a two necked round bottom flask fitted with reflux condenser and a separating funnel. Chloroacetyl chloride $8.75 \mathrm{ml}(0.11$ mol) was taken in dioxane ( $25 \mathrm{ml}$ approx), in the separating funnel. The chloroacetyl chloride was added in small portions to the vessel. After the complete addition, the contents of the flask was refluxed for an hour or so. After cooling the contents were poured on crushed ice. The precipitated product was filtered and washed several times with ice cold distilled water and recrystallized from absolute ethanol.

4-(chloroacyl amino)-3-mercapto-5-(4-methyl phenyl)-1,2,4-triazole. (5a). Yield 73\%, mp: 176- 
$178^{\circ} \mathrm{C}$. IR (KBr), v cm ${ }^{-1}: 1350(\mathrm{C}-\mathrm{N}), 1540(\mathrm{C}=\mathrm{N})$, 745 (C-Cl), 3000 (Ar-CH), 2550 (SH), 3305 (NH).

4-(chloro-acyl amino)-3-mercapto-5-(4-methoxyphenyl)-1,2,4-triazole. (5b). Yield 56\%, mp: 164$166^{\circ} \mathrm{C}$. IR (KBr), $v \mathrm{~cm}^{-1}: 1350(\mathrm{C}-\mathrm{N}), 1540(\mathrm{C}=\mathrm{N})$, 745 (C-Cl), 3000 (Ar-CH), $2550(\mathrm{SH}), 3305(\mathrm{NH})$, 1272, 1084 (C-O-C).

General procedure for title compounds (614a,b). Compound 4-(chloroacyl-amino)-3-mercapto-5(4-substituted) phenyl-1,2,4-triazole $\mathbf{5 a , b}(0.025 \mathrm{~mol})$ and respective amine $(0.030 \mathrm{~mol})$ along with the triethylamine $(0.030 \mathrm{~mol})$ were taken in a round bottomed flask in benzene ( $75 \mathrm{ml}$ approx). The contents were refluxed for 3-4 hr. The precipitated triethylamine hydrochloride was separated out. The organic layer was washed several times with distilled water to remove last traces of hydrochloride. Benzene was distilled off under vacuum and the crude product was separated. Purification of the compounds was done by repeated crystallization from appropriate solvents and melting point was determined. Physico-chemical properties of these synthesized compounds are shown in Table 1.

6a: 4-(piperidin-1'yl) ethanoylamino-3mercapto-5-(4-methyl) phenyl-1,2,4-triazole. FT$\operatorname{IR}\left(\mathrm{KBr}, v \mathrm{~cm}^{-1}\right): 3100(\mathrm{Ar}-\mathrm{H}), 2949.9(\mathrm{C}-\mathrm{H}), 1800$ (C-C overtone), $2521(\mathrm{~S}-\mathrm{H}), 1660(\mathrm{C}=\mathrm{O}), 1370$ (CN), 1530 (C=N str), $3300(\mathrm{~N}-\mathrm{H}), 1617.1$ (Ar-C=C). ${ }^{1} \mathrm{H}$ NMR (DMSO-d $\left.\mathrm{d}_{6}, \delta \mathrm{ppm}\right): \delta 1.19-1.76(\mathrm{~m}, 10 \mathrm{H}$, piper.), 2.31 (s, $\left.3 \mathrm{H}, \mathrm{Ar}-\mathrm{CH}_{3}\right), 3.15$ (s, $2 \mathrm{H}, \mathrm{COCH}_{2}$ ), 7.13-7.15(d, $J=8.7,2 \mathrm{H}, \mathrm{Ar}-\mathrm{H}), 7.99-8.00$ (d, $J=8.5$, 2H, Ar-H), 9.87 (s, 1H, SH), 10.13 (s, 1H, CONH). ${ }^{13} \mathrm{C}-\mathrm{NMR}\left(\mathrm{DMSO}_{6}, \delta \mathrm{ppm}\right): \delta 163.5\left(\mathrm{C}_{3}\right.$ and $\mathrm{C}_{5}$ triazole $), 133,126,129.5,141.5,19.97\left(\mathrm{C}_{1}, \mathrm{C}_{2}\right.$, and $\mathrm{C}_{6}, \mathrm{C}_{3}$, and $\left.\mathrm{C}_{5}, \mathrm{C}_{4}, \mathrm{C}_{4}{ }^{\prime} \mathrm{a}\right), 178.4$ (C of NHCO), $58(\mathrm{C}$ of $\left.\mathrm{CH}_{2} \mathrm{~N}\right), 49.3,25.4$ and $27.86\left(\mathrm{C}_{\mathrm{a}}\right.$ and $\mathrm{C}_{\mathrm{e}}, \mathrm{C}_{\mathrm{b}}$ and $\mathrm{C}_{\mathrm{d}}$ $\mathrm{C}_{\mathrm{c}}$ of piperidine). FABMS $\mathrm{m} / \mathrm{z}: 331(\mathrm{M}+1)$.

7a: 4-(2'-methyl-piperidin-1'yl) ethanoylamino-3-mercapto-5-(4-methyl) phenyl-1,2,4triazole. FT-IR (KBr, $\left.v \mathrm{~cm}^{-1}\right): 3100(\mathrm{Ar}-\mathrm{H}), 2949.9$ (C-H), 1800 (C-C overtone), 2524 (S-H), 1660 $(\mathrm{C}=\mathrm{O}), 1387(\mathrm{C}-\mathrm{N}), 1588(\mathrm{C}=\mathrm{N}), 1380(\mathrm{C}-\mathrm{H}), 3350$ $(\mathrm{N}-\mathrm{H}) .{ }^{1} \mathrm{H}$ NMR (DMSO-d 6 , $\delta$ ppm): $\delta 1.13$ (s, 3H, $\mathrm{CH}_{3}$ - piper.), 1.31-2.56 (m, $14 \mathrm{H}, \mathrm{CH}, \mathrm{CH}_{2}, \& \mathrm{CH}_{3}$ ),
7.01-7.03 (d, $J=8.3,2 \mathrm{H}, \operatorname{Ar}-\mathrm{H}), 8.03-8.05$ (d, $J=8.9$, 2H, Ar-H), 9.67 (s, 1H, SH), 10.01 (s, 1H, CONH) ${ }^{13}$ C-NMR (DMSO-d ${ }_{6}, \delta$ ppm): $\delta 153\left(\mathrm{C}_{3}\right.$ and $\mathrm{C}_{5}$ triazole $), 133,126,129,141,19\left(\mathrm{C}_{1}\right.$, and $\mathrm{C}_{2}, \mathrm{C}_{6}$, and $\mathrm{C}_{3}, \mathrm{C}_{5}$, and $\mathrm{C}_{4}$, and $\mathrm{C}_{4}{ }^{\prime}$ ), 172 (C of $\left.\mathrm{NHCO}\right), 57(\mathrm{C}$ of $\left.\mathrm{CH}_{2} \mathrm{~N}\right), 44,30,29,22,50$ and $18\left(\mathrm{C}_{\mathrm{a}}, \mathrm{C}_{\mathrm{b}}, \mathrm{C}_{\mathrm{c}}, \mathrm{C}_{\mathrm{d}}, \mathrm{C}_{\mathrm{e}}\right.$ and $\mathrm{C}_{\mathrm{f}}$ of 2-Methyl piperidine). FABMS $\mathrm{m} / \mathrm{z}: 345$ $(\mathrm{M}+1)$.

8a: 4-(4'-methyl-piperidin-1'yl) ethanoylamino-3-mercapto-5-(4-methyl) phenyl-1,2,4-triazole. FT-IR (KBr, v cm $\left.{ }^{-1}\right): 3100$ (Ar-H), $2949.9(\mathrm{C}-\mathrm{H})$, 1850 (C-C overtone), 2550 (S-H), 1673 (C=O), 1412 $(\mathrm{C}-\mathrm{N}), 1540(\mathrm{C}=\mathrm{N}), 3300(\mathrm{~N}-\mathrm{H}), 1460(\mathrm{C}-\mathrm{H}) .{ }^{1} \mathrm{H}$ NMR (DMSO-d ${ }_{6}, \delta$ ppm): 1.11 (s, $3 \mathrm{H}, \mathrm{CH}_{3^{-}}$piper.), 1.38-2.49 (m, 14H, CH, $\mathrm{CH}_{2}$, \& $\left.\mathrm{CH}_{3}\right), 6.72-7.18(\mathrm{~m}$, 7H, Ar-H), 8.03-8.05 (d, J=8.8, 2H, Ar-H), 9.69 (s, 1H, SH), 10.08 (s, 1H, CONH). ${ }^{13} \mathrm{C}-\mathrm{NMR}$ (DMSO$\left.\mathrm{d}_{6}, \delta \mathrm{ppm}\right): 148\left(\mathrm{C}_{3}\right.$ and $\mathrm{C}_{5}$ triazole $), 129,121,126$, 141, $20\left(\mathrm{C}_{1}\right.$, and $\mathrm{C}_{2}, \mathrm{C}_{6}$, and $\mathrm{C}_{3}, \mathrm{C}_{5}$, and $\mathrm{C}_{4}$, and $\mathrm{C}_{4}{ }^{\prime} \mathrm{a}$, 178 (C of NHCO), $59\left(\mathrm{C}\right.$ of $\left.\mathrm{CH}_{2} \mathrm{~N}\right), 44,31,29,21$, 50 and $19\left(\mathrm{C}_{\mathrm{a}}, \mathrm{C}_{\mathrm{b}}, \mathrm{C}_{\mathrm{c}}, \mathrm{C}_{\mathrm{d}}, \mathrm{C}_{\mathrm{e}}\right.$ and $\mathrm{C}_{\mathrm{f}}$ of 2-Methyl piperidine).FABMS $m / z: 345(\mathrm{M}+1)$.

9a: 4-(2'-Ethyl-piperidin-1'yl)ethanoylamino3-mercapto-5-(4-methyl) phenyl-1,2,4-triazole. FTIR ( $\mathrm{KBr}, v \mathrm{~cm}^{-1}$ ): 3100 (Ar-H str), 2949.2 (C-H str), 1900 (C-C overtone), 2520 (S-H str), 1660 (C=O str), $1410(\mathrm{C}-\mathrm{N}), 1550$ (C=N str), $3350(\mathrm{~N}-\mathrm{H}), 1479$ (CH). ${ }^{1} \mathrm{H}$ NMR (DMSO-d 6 , $\delta$ ppm): 0.71-1.68 $(\mathrm{m}, 11 \mathrm{H}$, $\mathrm{CH}_{2}$ ), 2.78-2.89 (m, 6H, CH, $\mathrm{CH}_{2}$ \& $\mathrm{Ar}-\mathrm{CH}_{3}$ ), 7.117.13 (d, J=9.7, 2H, Ar-H), 8.40-8.42 (d, J=8.5, 2H, Ar-H), 9.91 (s, 1H, SH), 9.99 (s, 1H, CONH). ${ }^{13} \mathrm{C}-$ NMR (DMSO- $\left.\mathrm{d}_{6}, \delta \mathrm{ppm}\right): 142\left(\mathrm{C}_{3}\right.$ and $\mathrm{C}_{5}$ triazole), 133, 126, 129, 137, $21\left(\mathrm{C}_{1}\right.$, and $\mathrm{C}_{2}, \mathrm{C}_{6}$, and $\mathrm{C}_{3}, \mathrm{C}_{5}$, and $\mathrm{C}_{4}$, and $\mathrm{C}_{4}{ }^{\prime}$ ) $, 169(\mathrm{C}$ of $\mathrm{NHCO}), 57\left(\mathrm{C}_{\text {of }} \mathrm{CH}_{2} \mathrm{~N}\right)$, 58, 34, 22, 26, and $44\left(\mathrm{C}_{\mathrm{a}}\right.$ and $\mathrm{C}_{\mathrm{b}}, \mathrm{C}_{\mathrm{c}}$ and $\mathrm{C}_{\mathrm{d}}$ and $\mathrm{C}_{\mathrm{e}}$ of 2-Ethyl piperidine), 9 ( $\mathrm{C}_{\mathrm{g}}$ of methyl group of 2Ethyl piperidine). FABMS $m / z: 359(\mathrm{M}+1)$.

10a: 4-(4'-benzyl-piperidin-1'yl) ethanoylamino-3-mercapto-5-(4-methyl) phenyl-1,2,4-triazole. FT-IR (KBr, v cm $\left.{ }^{-1}\right): 3024$ (Ar-H), 1800 (C-C overtone), $2530(\mathrm{~S}-\mathrm{H}), 1670$ (C=O str), 1617 (Ar$\mathrm{C}=\mathrm{C}), 1360 \quad(\mathrm{C}-\mathrm{N}), 1540 \quad(\mathrm{C}=\mathrm{N}$ str$), 1499(\mathrm{C}-\mathrm{H})$, $3340(\mathrm{~N}-\mathrm{H}) .{ }^{1} \mathrm{H}$ NMR (DMSO-d $\left.{ }_{6}, \delta \mathrm{ppm}\right):$ 1.05-1.76 $\left(\mathrm{m}, 11 \mathrm{H}, \mathrm{CH}_{2}\right), 2.39$ (s, 3H, Ar- $\left.\mathrm{CH}_{3}\right), 3.10(\mathrm{~s}, 2 \mathrm{H}$, 
$\left.\mathrm{COCH}_{2}\right), \quad 6.72-7.18(\mathrm{~m}, 7 \mathrm{H}, \mathrm{Ar}-\mathrm{H}), 7.71-7.73(\mathrm{~d}$, $J=7.9,2 \mathrm{H}$, Ar-H), 8.99 (s, 1H, SH), 9.87 (s, 1H, CONH). ${ }^{13} \mathrm{C}-\mathrm{NMR}$ (DMSO-d 6 , $\left.\delta \mathrm{ppm}\right): 148\left(\mathrm{C}_{3}\right.$ and $\mathrm{C}_{5}$ triazole $), 133,126,129,137,21\left(\mathrm{C}_{1}\right.$, and $\mathrm{C}_{2}, \mathrm{C}_{6}$, and $\mathrm{C}_{3}, \mathrm{C}_{5}$, and $\mathrm{C}_{4}$, and $\left.\mathrm{C}_{4}{ }^{\prime} \mathrm{a}\right), 172(\mathrm{C}$ of $\mathrm{NHCO}), 58(\mathrm{C}$ of $\left.\mathrm{CH}_{2} \mathrm{~N}\right), 44,28,36\left(\mathrm{C}_{\mathrm{a}}, \mathrm{C}_{\mathrm{e}}\right.$ and $\mathrm{C}_{\mathrm{b}}, \mathrm{C}_{\mathrm{d}}$ and $\mathrm{C}_{\mathrm{c}}$ of piperidine) $42.3\left(\mathrm{C}_{\mathrm{f}}\right.$ of benzyl piperidine $), 139$,

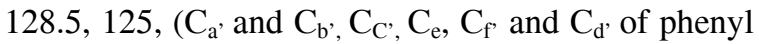
ring of benzyl piperidine). FABMS $m / z: 421(\mathrm{M}+1)$.

11a: 4-(Morpholin-1'-yl) ethanoylamino-3mercapto-5-(4-methyl) phenyl-1,2,4-triazole. FTIR $\left(\mathrm{KBr}, v \mathrm{~cm}^{-1}\right): 1410(\mathrm{C}-\mathrm{N}), 1540(\mathrm{C}=\mathrm{N}), 3100(\mathrm{Ar}-$ $\mathrm{H}$ ), 1850 (C-C overtone), 2540 (S-H str), 1660 $(\mathrm{C}=\mathrm{O}), 1068$ (C-O), 3360 (N-H). ${ }^{1} \mathrm{H}$ NMR (DMSO$\mathrm{d}_{6}, \delta \mathrm{ppm}$ ): 2.36-2.59 (t, 2x2H, $\mathrm{CH}_{2}$-morph.), 2.412.69 (m, 7H, $\mathrm{CH}_{2}$-morph. \& Ar- $\left.\mathrm{CH}_{3}\right), 3.13$ (s, 2H, $\mathrm{COCH}_{2}$ ), 7.16-7.18 (d, $\left.J=10.0,2 \mathrm{H}, \mathrm{Ar}-\mathrm{H}\right), 8.15-8.17$ (d, J=9.8, 2H, Ar-H), 9.89 (s, 1H, SH), 10.07 (s, 1H, CONH). ${ }^{13} \mathrm{C}-\mathrm{NMR}$ (DMSO-d $\left.\mathrm{d}_{6}, \delta \mathrm{ppm}\right): 138\left(\mathrm{C}_{3}\right.$ and $\mathrm{C}_{5}$ triazole $), 133,126,130,142,22\left(\mathrm{C}_{1}\right.$, and $\mathrm{C}_{2}, \mathrm{C}_{6}$, and $\mathrm{C}_{3}, \mathrm{C}_{5}$, and $\mathrm{C}_{4}$, and $\mathrm{C}_{4}{ }^{\prime}$ ), 166 (C of $\left.\mathrm{NHCO}\right), 67(\mathrm{C}$ of $\left.\mathrm{CH}_{2} \mathrm{~N}\right), 65,71\left(\mathrm{C}_{\mathrm{a}}, \mathrm{C}_{\mathrm{e}}\right.$ and $\mathrm{C}_{\mathrm{b}}, \mathrm{C}_{\mathrm{d}}$ of morpholine $)$. FABMS $m / z: 333(\mathrm{M}+1)$.

12a: 4-(pyrrolidin-1'yl) ethanoylamino-3mercapto-5-(4-methyl) phenyl-1,2,4-triazole. FTIR $\left(\mathrm{KBr}, v \mathrm{~cm}^{-1}\right): 1378(\mathrm{C}-\mathrm{N}), 1550(\mathrm{C}=\mathrm{N}), 3050$ (Ar-H), 2920.1 (C-H), 1850 (C-C overtone), 2541 (S$\mathrm{H}), 1660(\mathrm{C}=\mathrm{O}), 1618(\mathrm{Ar}-\mathrm{C}=\mathrm{C}), 1507(\mathrm{C}-\mathrm{H}) .{ }^{1} \mathrm{H}$ NMR (DMSO-d $6, \delta$ ppm): 1.09-1.17 (t, 2x2H, $\mathrm{CH}_{2^{-}}$ pyrr.), 1.19-1.24 (t, 2x2H, $\mathrm{CH}_{2}$-pyrr.), 2.36 (s, 3H, Ar- $\mathrm{CH}_{3}$ ), 3.10 (s, 2H, $\mathrm{COCH}_{2}$ ), 7.09-7.11 (d, J=9.9, $2 \mathrm{H}, \mathrm{Ar}-\mathrm{H}$ ), 8.10-8.12 (d, J=8.9, 2H, Ar-H), 9.78 (s, $1 \mathrm{H}, \mathrm{SH}), 10.13$ (s, 1H, CONH). ${ }^{13} \mathrm{C}-\mathrm{NMR}$ (DMSO$\left.\mathrm{d}_{6} \delta \mathrm{ppm}\right): 144\left(\mathrm{C}_{3}\right.$ and $\mathrm{C}_{5}$ triazole $), 133,126.1,130$, 137, $22\left(\mathrm{C}_{1}\right.$, and $\mathrm{C}_{2}, \mathrm{C}_{6}$, and $\mathrm{C}_{3}, \mathrm{C}_{5}$, and $\mathrm{C}_{4}$, and $\mathrm{C}_{4}{ }^{\prime} \mathrm{a}$, $171(\mathrm{C}$ of $\mathrm{NHCO}), 66\left(\mathrm{C}\right.$ of $\left.\mathrm{CH}_{2} \mathrm{~N}\right), 52,23\left(\mathrm{C}_{\mathrm{a}^{\prime}}, \mathrm{C}_{\mathrm{d}^{\prime}}\right.$ and $\mathrm{C}_{\mathrm{b}}, \mathrm{C}_{\mathrm{c}}$, of pyrrolidine). FABMS m/z: 317 $(\mathrm{M}+1)$.

13a: 4-(pyrrolidin-2'one-1'yl) ethanoylamino3-mercapto-5-(4-methyl) phenyl-1,2,4-triazole. FTIR $\left(\mathrm{KBr}, v \mathrm{~cm}^{-1}\right): 1410(\mathrm{C}-\mathrm{N}), 1530(\mathrm{C}=\mathrm{N}), 3000$ (Ar-H), 2920 (C-H), 1850 (C-C overtone), 2520 (S$\mathrm{H}), 1660(\mathrm{C}=\mathrm{O}), 1700(\mathrm{C}=\mathrm{O} \gamma$-butyrolactam $) .{ }^{1} \mathrm{H}$ NMR (DMSO-d $\left.\mathrm{d}_{6}, \delta \mathrm{ppm}\right): \quad 2.06-2.41\left(\mathrm{~m}, 7 \mathrm{H}, \mathrm{CH}_{2^{-}}\right.$ pyrr. \& $\left.\mathrm{Ar}-\mathrm{CH}_{3}\right), 3.49-3.68\left(\mathrm{~m}, 4 \mathrm{H}, \mathrm{CH}_{2}\right.$-pyrr. \& $\mathrm{COCH}_{2}$ ), 7.19-7.21 (d, J=9.1, 2H, Ar-H), 8.23-8.25 (d, J=9.5, 2H, Ar-H), 9.68 (s, 1H, SH), 10.13 (s, 1H, CONH). ${ }^{13} \mathrm{C}-\mathrm{NMR}$ (DMSO-d $\left.\mathrm{d}_{6}, \delta \mathrm{ppm}\right): 148\left(\mathrm{C}_{3}\right.$ and $\mathrm{C}_{5}$ triazole $), 133,126,129,142,21\left(\mathrm{C}_{1}\right.$, and $\mathrm{C}_{2}, \mathrm{C}_{6}$, and $\mathrm{C}_{3}, \mathrm{C}_{5}$, and $\mathrm{C}_{4}$, and $\mathrm{C}_{4}$ 'a respectively), 178 (C of $\mathrm{NHCO}), 52\left(\mathrm{C}\right.$ of $\left.\mathrm{CH}_{2} \mathrm{~N}\right), 73,30,17$, and $43\left(\mathrm{C}_{\mathrm{a}}\right.$, and $\mathrm{C}_{\mathrm{b}}$ and $\mathrm{C}_{\mathrm{c}}$ and $\mathrm{C}_{\mathrm{d}}$ of pyrrolidinone respectively). FABMS $m / z: 331(\mathrm{M}+1)$.

14a: 4-(1'-Benzyl-piperazin-1'yl) ethanoylamino-3-mercapto-5-(4-methyl) phenyl-1,2,4triazole. FT-IR (KBr, $\left.v \mathrm{~cm}^{-1}\right): 1370(\mathrm{C}-\mathrm{N}), 1540$ $(\mathrm{C}=\mathrm{N}), 3100 \quad(\mathrm{Ar}-\mathrm{H}), 2925 \quad(\mathrm{C}-\mathrm{H}), 1800 \quad(\mathrm{C}-\mathrm{C}$ overtone), $2541(\mathrm{~S}-\mathrm{H}), 1660(\mathrm{C}=\mathrm{O}), 3436(\mathrm{~N}-\mathrm{H})$, 1074 (C-H piperazine), 1597 ( $\mathrm{Ar}-\mathrm{C}=\mathrm{C}$ ). ${ }^{1} \mathrm{H}$ NMR (DMSO-d ${ }_{6}, \delta$ ppm): $\delta \quad 2.36-2.89\left(\mathrm{~m}, 11 \mathrm{H}, \mathrm{CH}_{2^{-}}\right.$ piperazin \& $\left.\mathrm{Ar}-\mathrm{CH}_{3}\right), 3.14\left(\mathrm{~s}, 2 \mathrm{H}, \mathrm{COCH}_{2}\right), 3.59$ (s, 2H, Ar- $\mathrm{CH}_{2}$ ), 7.01-7.58 (m, 7H, Ar-H), 7.99-8.01 (d, $J=8.3,2 \mathrm{H}, \mathrm{Ar}-\mathrm{H}), 8.61(\mathrm{~s}, 1 \mathrm{H}, \mathrm{SH}), 9.95(\mathrm{~s}, 1 \mathrm{H}$, CONH). ${ }^{13} \mathrm{C}-\mathrm{NMR}$ (DMSO-d $\left.\mathrm{d}_{6}, \delta \mathrm{ppm}\right): 143.5\left(\mathrm{C}_{3}\right.$ and $\mathrm{C}_{5}$ triazole $), 133,126.1,129.9,137,21.8\left(\mathrm{C}_{1}\right.$, and $\mathrm{C}_{2}, \mathrm{C}_{6}$, and $\mathrm{C}_{3}, \mathrm{C}_{5}$, and $\mathrm{C}_{4}$, and $\mathrm{C}_{4}{ }^{\prime}$ ), 171 ( $\mathrm{C}$ of $\mathrm{NHCO}), 59.3\left(\mathrm{C}\right.$ of $\left.\mathrm{CH}_{2} \mathrm{~N}\right), 60,55\left(\mathrm{C}_{\mathrm{a}}, \mathrm{C}_{\mathrm{e}}\right.$ and $\mathrm{C}_{\mathrm{b}}, \mathrm{C}_{\mathrm{d}}$ of piperazine) $59\left(\mathrm{C}_{\mathrm{f}}\right.$ of benzyl), 136, 129, 128, 127 $\left(\mathrm{C}_{\mathrm{a}}\right.$, and $\mathrm{C}_{\mathrm{b}}, \mathrm{C}_{\mathrm{f}}$, and $\mathrm{C}_{\mathrm{c}}, \mathrm{C}_{\mathrm{e}}$, and $\mathrm{C}_{\mathrm{d}}$, of phenyl ring of benzyl piperazine). FABMS $m / z: 422(\mathrm{M}+1)$.

6b: 4-(piperidin-1'yl) ethanoylamino-3mercapto-5-(4-methoxy) phenyl-1,2,4-triazole. FTIR $\left(\mathrm{KBr}, v \mathrm{~cm}^{-1}\right): 691(\mathrm{C}-\mathrm{H}), 1519(\mathrm{C}=\mathrm{N}), 3314(\mathrm{~N}-$ H), 3050 (Ar-H), 2840 (C-H Anisole), 1251 (C-O-C asymm.), 1072 (C-O-C symm.), 2520 (S-H str), 1714 $(\mathrm{C}=\mathrm{O}), 1467$ (CH-Piper.). ${ }^{1} \mathrm{H}$ NMR (DMSO-d ${ }_{6}, \delta$ ppm): 1.19-1.76 (m, 10H, piper.), 3.15 (s, 2H, $\mathrm{COCH}_{2}$ ), 3.75 (s, 3H, Ar- $\mathrm{OCH}_{3}$ ), 7.11 (d, 2H, Ar-H), 7.90 (d, 2H, Ar-H), 9.81 (s, 1H, SH), 10.08 (s, 1H, CONH). ${ }^{13} \mathrm{C}-\mathrm{NMR}$ (DMSO-d $\left.{ }_{6}, \delta \mathrm{ppm}\right): 161\left(\mathrm{C}_{3}\right.$ and $\mathrm{C}_{5}$ triazole $), 128,127,115,163,56\left(\mathrm{C}_{1}\right.$, and $\mathrm{C}_{2}, \mathrm{C}_{6}$, and $\mathrm{C}_{3}, \mathrm{C}_{5}$, and $\mathrm{C}_{4}$, and $\left.\mathrm{C}_{4}{ }^{\prime} \mathrm{a}\right), 178(\mathrm{C}$ of $\mathrm{NHCO}), 59(\mathrm{C}$ of $\left.\mathrm{CH}_{2} \mathrm{~N}\right), 53,24$ and $26\left(\mathrm{C}_{\mathrm{a}}, \mathrm{C}_{\mathrm{e}}\right.$, and $\mathrm{C}_{\mathrm{b}}, \mathrm{C}_{\mathrm{d}}$ and $\mathrm{C}_{\mathrm{c}}$ of piperidine). FABMS $m / z: 347(\mathrm{M}+1)$.

7b: 4-(2'-methyl-piperidin-1'yl) ethanoylamino-3-mercapto-5-(4-methoxy) phenyl-1,2,4-triazole. FT-IR (KBr, $\left.v \mathrm{~cm}^{-1}\right)$ : $692(\mathrm{C}-\mathrm{H}), 1350(\mathrm{C}-\mathrm{N}), 1516$ $(\mathrm{C}=\mathrm{N}), 3050$ (Ar-H str), 2947 (C-H Anisole), 1252 
(C-O-C asymm.), 1073 (C-O-C symm.), 2520 (S-H), 1719 (C=O), 1611 (C=C Ar.), 1440 (CH-Piper.). ${ }^{1} \mathrm{H}$ NMR (DMSO-d $\mathrm{d}_{6}, \delta \mathrm{ppm}$ ): 1.13 (s, $3 \mathrm{H}, \mathrm{CH}_{3}-$ piper.), 1.31-2.56 (m, $\left.11 \mathrm{H}, \mathrm{CH}, \mathrm{CH}_{2}\right), 3.72(\mathrm{~s}, 3 \mathrm{H}, \mathrm{Ar}-$ $\left.\mathrm{OCH}_{3}\right), 7.06(\mathrm{~d}, 2 \mathrm{H}, \mathrm{Ar}-\mathrm{H}), 8.10(\mathrm{~d}, 2 \mathrm{H}, \mathrm{Ar}-\mathrm{H}), 9.76$ (s, 1H, SH), 10.05 (s, 1H, CONH). ${ }^{13} \mathrm{C}-\mathrm{NMR}$ (DMSO- $\left.\mathrm{d}_{6}, \delta \mathrm{ppm}\right): 161\left(\mathrm{C}_{3}\right.$ and $\mathrm{C}_{5}$ triazole), 129, 128, 115, 163, $56\left(\mathrm{C}_{1}\right.$, and $\mathrm{C}_{2}, \mathrm{C}_{6}$, and $\mathrm{C}_{3}, \mathrm{C}_{5}$, and $\mathrm{C}_{4}$, and $\mathrm{C}_{4}{ }^{\prime}$ ) $, 169(\mathrm{C}$ of $\mathrm{NHCO}), 56\left(\mathrm{C}\right.$ of $\left.\mathrm{CH}_{2} \mathrm{~N}\right), 41,19$, 35,23 and $26\left(C_{a}\right.$ and $C_{b}$, and $C_{c}$, and $C_{d}$ and $C_{e}$ of 2Methyl piperidine $), 39\left(\mathrm{C}_{\mathrm{f}}\right.$ of methyl group). FABMS $m / z: 361(\mathrm{M}+1)$.

8b: 4-(4'-methyl-piperidin-1'yl) ethanoylamino-3-mercapto-5-(4-methoxy) phenyl-1,2,4-triazole. FT-IR (KBr, v cm $\left.{ }^{-1}\right): 699$ (C-H), 1351 (C-N), 1525 $(\mathrm{C}=\mathrm{N}) 3050$ (Ar-H), 2835 (C-H anisole), 1254 (C-OC asymm.), 1073 (C-O-C symm.), 2554 (S-H), 1660 $(\mathrm{C}=\mathrm{O}), 1467$ (CH-Piper.). ${ }^{1} \mathrm{H}$ NMR (DMSO-d ${ }_{6}, \delta$ ppm): 1.14 (s, $3 \mathrm{H}, \mathrm{CH}_{3}$ - piper.), 1.34-2.45 (m, $11 \mathrm{H}$, $\left.\mathrm{CH} \& \mathrm{CH}_{2}\right), 3.88\left(\mathrm{~s}, 3 \mathrm{H}, \mathrm{Ar}-\mathrm{OCH}_{3}\right)$ 6.74-7.20 (m, 7H, Ar-H), 8.08 (d, 2H, Ar-H), 9.71 (s, 1H, SH), 10.03 (s, 1H, CONH). ${ }^{13} \mathrm{C}-\mathrm{NMR}$ (DMSO-d 6 , $\delta$ ppm): $170\left(\mathrm{C}_{3}\right.$ and $\mathrm{C}_{5}$ triazole $), 129,128,115,163,56\left(\mathrm{C}_{1}\right.$, and $\mathrm{C}_{2}, \mathrm{C}_{6}$, and $\mathrm{C}_{3}, \mathrm{C}_{5}$, and $\mathrm{C}_{4}$, and $\mathrm{C}_{4}{ }^{\prime}$ ), 178 (C of NHCO $), 55\left(\mathrm{C}\right.$ of $\left.\mathrm{CH}_{2} \mathrm{~N}\right), 50,32,26$ and $20\left(\mathrm{C}_{\mathrm{a}}, \mathrm{C}_{\mathrm{e}}\right.$, and $\mathrm{C}_{\mathrm{b}}, \mathrm{C}_{\mathrm{d}}$ and $\mathrm{C}_{\mathrm{c}}$ and $\mathrm{C}_{\mathrm{f}}$ of 4-Methyl piperidine). FABMS $m / z: 361(\mathrm{M}+1)$.

9b: 4-(2'-Ethyl-piperidin-1'yl) ethanoylamino3-mercapto-5-(4-methoxy) phenyl-1,2,4-triazole. FT-IR (KBr, $\left.v \mathrm{~cm}^{-1}\right): 693$ (C-H), 1351 (C-N), 1551 (C=N str), 3046 (Ar-H), 2840 (C-H Anisole), 1253 (C-O-C asymm.), 1068 (C-O-C symm.), 2554 (S-H), 1660 (C=O), 3315 (N-H), 1467 (CH-Piper.). ${ }^{1} \mathrm{H}$ NMR (DMSO-d 6 , $\delta$ ppm): 0.74-1.71 $\left(\mathrm{m}, 11 \mathrm{H}, \mathrm{CH}_{2}\right)$, 2.76-2.87 (m, 3H, CH \& $\left.\mathrm{CH}_{2}\right), 3.79(\mathrm{~s}, 3 \mathrm{H}, \mathrm{Ar}-$ $\left.\mathrm{OCH}_{3}\right), 7.08$ (d, 2H, Ar-H), 8.38 (d, 2H, Ar-H), 9.89 (s, 1H, SH), 9.98 (s, 1H, CONH). ${ }^{13} \mathrm{C}-\mathrm{NMR}$ (DMSO$\left.\mathrm{d}_{6}, \delta \mathrm{ppm}\right): 161\left(\mathrm{C}_{3}\right.$ and $\mathrm{C}_{5}$ triazole $), 129,128,115$, 163, $56\left(\mathrm{C}_{1}\right.$, and $\mathrm{C}_{2}, \mathrm{C}_{6}$, and $\mathrm{C}_{3^{\prime}}, \mathrm{C}_{5}$, and $\mathrm{C}_{4}$, and $\left.\mathrm{C}_{4}{ }^{\prime} \mathrm{a}\right)$, 178 (C of NHCO), $56\left(\mathrm{C}\right.$ of $\left.\mathrm{CH}_{2} \mathrm{~N}\right), 57,29,24,25$ and $50\left(\mathrm{C}_{\mathrm{a}}\right.$ and $\mathrm{C}_{\mathrm{b}}$, and $\mathrm{C}_{\mathrm{c}}$, and $\mathrm{C}_{\mathrm{d}}$ and $\mathrm{C}_{\mathrm{e}}$ of 2-Ethyl piperidine), 27 ( $\mathrm{C}_{\mathrm{f}}$ of methylene group), $9\left(\mathrm{C}_{\mathrm{g}}\right.$ of methyl group). FABMS m/z: $375(\mathrm{M}+1)$. 10b: 4-(4'-benzyl-piperidin-1'yl) ethanoylamino-3-mercapto-5-(4-methoxy) phenyl-1,2,4-triazole. FT-IR (KBr, $\left.v \mathrm{~cm}^{-1}\right)$ : $692(\mathrm{C}-\mathrm{H}), 1350(\mathrm{C}-\mathrm{N}), 1519$ $(\mathrm{C}=\mathrm{N}), 3050(\mathrm{Ar}-\mathrm{H}), 2981$ (C-H Anisole), 1251 (CO-C asymm.), 1072 (C-O-C symm.), 2520 (S-H), $1716(\mathrm{C}=\mathrm{O}), 1466$ (CH-Piper.). ${ }^{1} \mathrm{H}$ NMR (DMSO-d $\mathrm{d}_{6}$, $\delta \mathrm{ppm}): 1.05-1.76\left(\mathrm{~m}, 11 \mathrm{H}, \mathrm{CH}_{2}\right), 3.59(\mathrm{~s}, 3 \mathrm{H}, \mathrm{Ar}-$ $\left.\mathrm{OCH}_{3}\right), 3.14\left(\mathrm{~s}, 2 \mathrm{H}, \mathrm{COCH}_{2}\right), 6.75-7.21(\mathrm{~m}, 7 \mathrm{H}, \mathrm{Ar}-$ H), 7.66 (d, 2H, Ar-H), 8.97 (s, 1H, SH), 9.93 (s, 1H, CONH). ${ }^{13} \mathrm{C}-\mathrm{NMR}$ (DMSO-d $\left.\mathrm{d}_{6}, \delta \mathrm{ppm}\right): 161\left(\mathrm{C}_{3}\right.$ and $\mathrm{C}_{5}$ triazole $), 129,128,115,163,56\left(\mathrm{C}_{1}\right.$, and $\mathrm{C}_{2}, \mathrm{C}_{6}$. and $\mathrm{C}_{3}, \mathrm{C}_{5}$, and $\mathrm{C}_{4}$, and $\mathrm{C}_{4}$ 'a respectively), 178 ( $\mathrm{C}$ of $\mathrm{NHCO}), 60\left(\mathrm{C}\right.$ of $\left.\mathrm{CH}_{2} \mathrm{~N}\right), 50,29$ and $32\left(\mathrm{C}_{\mathrm{a}}, \mathrm{C}_{\mathrm{e}}\right.$ and $\mathrm{C}_{\mathrm{b}}, \mathrm{C}_{\mathrm{d}}$ and $\mathrm{C}_{\mathrm{c}}$ of piperidine respectively $), 40\left(\mathrm{C}_{\mathrm{f}}\right.$ of benzyl-piperidine), 139, 124 and $126\left(\mathrm{C}_{\mathrm{a}}\right.$ and $\mathrm{C}_{\mathrm{b}^{\prime}}, \mathrm{C}_{\mathrm{C}^{\prime}}$, $\mathrm{C}_{\mathrm{e}}, \mathrm{C}_{\mathrm{f}}$, and $\mathrm{C}_{\mathrm{d}}$, of benzyl-piperidine). FABMS $\mathrm{m} / \mathrm{z}$ : $437(\mathrm{M}+1)$.

11b: 4-(Morpholin-1'-yl) ethanoylamino-3mercapto-5-(4-methoxy) phenyl-1,2,4-triazole. FTIR $\left(\mathrm{KBr}, v \mathrm{~cm}^{-1}\right): 699(\mathrm{C}-\mathrm{H}), 1352(\mathrm{C}-\mathrm{N}), 1522$ $(\mathrm{C}=\mathrm{N}), 3100(\mathrm{Ar}-\mathrm{H}), 2970$ (C-H Anisole), 1253 (CO-C asymm.), 1040 (C-O-C symm.), 2520 (S-H), $1660(\mathrm{C}=\mathrm{O}$ ), 1060 (C-O str. of cyclic ether), 1501 (CH-Morph.), 1615 (C=C Ar.). ${ }^{1} \mathrm{H}$ NMR (DMSO-d ${ }_{6}$, $\delta \mathrm{ppm}$ ): $2.33-2.56$ (t, $2 \times 2 \mathrm{H}, \mathrm{CH}_{2}$-morph.), 2.38-2.66 (m, 4H, $\mathrm{CH}_{2}$-morph.), 3.13 (s, 2H, $\mathrm{COCH}_{2}$ ), 3.85 (s, $\left.3 \mathrm{H}, \mathrm{Ar}-\mathrm{OCH}_{3}\right) 7.14$ (d, 2H, Ar-H), 8.11 (d, 2H, Ar$\mathrm{H}), 9.92(\mathrm{~s}, 1 \mathrm{H}, \mathrm{SH}), 10.11(\mathrm{~s}, 1 \mathrm{H}, \mathrm{CONH}) .{ }^{13} \mathrm{C}-$ NMR (DMSO- $\left.\mathrm{d}_{6}, \delta \mathrm{ppm}\right): 161\left(\mathrm{C}_{3}\right.$ and $\mathrm{C}_{5}$ triazole), $129,128,115,163,56\left(\mathrm{C}_{1}\right.$, and $\mathrm{C}_{2}, \mathrm{C}_{6}$, and $\mathrm{C}_{3}, \mathrm{C}_{5}$, and $\mathrm{C}_{4}$, and $\mathrm{C}_{4}{ }^{\prime}$ a respectively), 178 (C of $\mathrm{NHCO}$ ), 60 (C of $\left.\mathrm{CH}_{2} \mathrm{~N}\right), 57$ and $72\left(\mathrm{C}_{\mathrm{a}}, \mathrm{C}_{\mathrm{e}}\right.$ and $\mathrm{C}_{\mathrm{b}}, \mathrm{C}_{\mathrm{d}}$ of morpholine respectively). FABMS m/z: $349(\mathrm{M}+1)$.

12b: 4-(pyrrolidin-1'yl) ethanoylamino-3mercapto-5-(4-methoxy) phenyl-1,2,4-triazole. FTIR $\left(\mathrm{KBr}, v \quad \mathrm{~cm}^{-1}\right): 699(\mathrm{C}-\mathrm{H}), 1352(\mathrm{C}-\mathrm{N}), 1530$ $(\mathrm{C}=\mathrm{N}), 3098$ (Ar-H), 2981 (C-H Anisole), 1250 (CO-C asymm.), 1080 (C-O-C symm.), 2578 (S-H), 1660 (C=O), 3350 (N-H), 1516 (CH-Pyrrol.), 1615 (C=C Ar.). ${ }^{1} \mathrm{H}$ NMR (DMSO-d ${ }_{6}, \delta$ ppm): 1.08-1.16 (t, $2 \times 2 \mathrm{H}, \mathrm{CH}_{2}$-pyrr.), 1.21-1.26 (t, $2 \times 2 \mathrm{H}, \mathrm{CH}_{2}$-pyrr.), 3.10 (s, 2H, $\mathrm{COCH}_{2}$ ), 3.86 (s, 3H, $\left.\mathrm{Ar}-\mathrm{OCH}_{3}\right), 7.09$ (d, $2 \mathrm{H}, \operatorname{Ar}-\mathrm{H}), 8.13(\mathrm{~d}, 2 \mathrm{H}, \operatorname{Ar}-\mathrm{H}), 9.81(\mathrm{~s}, 1 \mathrm{H}, \mathrm{SH})$, 10.16 (s, $1 \mathrm{H}, \mathrm{CONH}) .{ }^{13} \mathrm{C}-\mathrm{NMR}$ (DMSO-d ${ }_{6}, \delta \mathrm{ppm}$ ): 
$161\left(\mathrm{C}_{3}\right.$ and $\mathrm{C}_{5}$ triazole $), 129,128,115,163,56\left(\mathrm{C}_{1}\right.$, and $\mathrm{C}_{2}, \mathrm{C}_{6}$, and $\mathrm{C}_{3}, \mathrm{C}_{5}$, and $\mathrm{C}_{4}$, and $\mathrm{C}_{4}{ }^{\prime}$, respectively), $178(\mathrm{C}$ of $\mathrm{NHCO}), 59\left(\mathrm{C}\right.$ of $\left.\mathrm{CH}_{2} \mathrm{~N}\right), 51,23\left(\mathrm{C}_{\mathrm{a}}, \mathrm{C}_{\mathrm{d}^{\prime}}\right.$ and $\mathrm{C}_{\mathrm{b}}, \mathrm{C}_{\mathrm{c}}$, of pyrrolidine respectively). FABMS $\mathrm{m} / \mathrm{z}$ : $333(\mathrm{M}+1)$.

13b: 4-(pyrrolidin-2'one-1'yl) ethanoylamino3-mercapto-5-(4-methoxy) phenyl-1,2,4-triazole.

FT-IR (KBr, v cm $\left.{ }^{-1}\right): 693(\mathrm{C}-\mathrm{H}), 1351(\mathrm{C}-\mathrm{N}), 1524$ $(\mathrm{C}=\mathrm{N}), 3050(\mathrm{Ar}-\mathrm{H}), 2950$ (C-H Anisole), 1251 (CO-C asymm.), 1077 (C-O-C symm.), 2520 (S-H), $1660(\mathrm{C}=\mathrm{O}), 1700(\mathrm{C}=\mathrm{O} \gamma$-butyrolactam $), 1510(\mathrm{C}-\mathrm{H}$ Pyrrol.). ${ }^{1} \mathrm{H}$ NMR (DMSO-d $\left.\mathrm{d}_{6}, \delta \mathrm{ppm}\right)$ : 2.07-2.42 (m, 7H, $\mathrm{CH}_{2}$-pyrr.), 3.45-3.64 (m, 4H, $\mathrm{CH}_{2}$-pyrr. \& $\mathrm{COCH}_{2}$ ), 3.91 (s, 3H, Ar-OCH $\left.{ }_{3}\right), 7.18$ (d, 2H, Ar-H), 8.22 (d, 2H, Ar-H), 9.73 (s, 1H, SH), 10.09 (s, 1H, CONH). ${ }^{13} \mathrm{C}-\mathrm{NMR}$ (DMSO-d $\left.\mathrm{d}_{6}, \delta \mathrm{ppm}\right): 167\left(\mathrm{C}_{3}\right.$ and $\mathrm{C}_{5}$ triazole $), 129,128,115,163,56\left(\mathrm{C}_{1}\right.$, and $\mathrm{C}_{2}, \mathrm{C}_{6}$. and $\mathrm{C}_{3}, \mathrm{C}_{5}$, and $\mathrm{C}_{4}$, and $\mathrm{C}_{4}$ 'a respectively), 178 (C of $\mathrm{NHCO}), 53\left(\mathrm{C}\right.$ of $\left.\mathrm{CH}_{2} \mathrm{~N}\right), 177,36,16,40\left(\mathrm{C}_{\mathrm{a}}\right.$ and $\mathrm{C}_{\mathrm{b}}$ and $\mathrm{C}_{\mathrm{c}}$, and $\mathrm{C}_{\mathrm{d}}$ of pyrrolidine respectively). FABMS $m / z: 347(\mathrm{M}+1)$.

14b: 4-(1'-Benzyl-piperazin-1'yl) ethanoylamino-3-mercapto-5-(4-methoxy) phenyl-1,2,4-triazole. FT-IR (KBr, v cm $\left.{ }^{-1}\right): 694(\mathrm{C}-\mathrm{H}), 1352(\mathrm{C}-\mathrm{N}), 1522$ $(\mathrm{C}=\mathrm{N}), 3100$ (Ar-H), 2980 (C-H Anisole), 1253 (CO-C asymm.), 1079 (C-O-C symm.), 1070 (C-H piperazine) $2520(\mathrm{~S}-\mathrm{H}), 1670(\mathrm{C}=\mathrm{O}), 3367(\mathrm{~N}-\mathrm{H})$, 1466 (CH-Piper.). 2.37-2.90 (m, 8H, $\mathrm{CH}_{2}$-piperazin). ${ }^{1} \mathrm{H}$ NMR (DMSO-d $\left.{ }_{6}, \delta \mathrm{ppm}\right): 3.16\left(\mathrm{~s}, 2 \mathrm{H}, \mathrm{COCH}_{2}\right.$ ), 3.62 (s, $\left.2 \mathrm{H}, \mathrm{Ar}-\mathrm{CH}_{2}\right), 3.84$ (s, 3H, Ar- $\left.\mathrm{OCH}_{3}\right), 7.05-$ 7.62 (m, 7H, Ar-H), 7.98 (d, 2H, Ar-H), 8.63 (s, 1H, $\mathrm{SH}), 9.98(\mathrm{~s}, 1 \mathrm{H}, \mathrm{CONH}) .{ }^{13} \mathrm{C}-\mathrm{NMR}$ (DMSO-d $\mathrm{d}_{6}, \delta$ ppm): $161\left(\mathrm{C}_{3}\right.$ and $\mathrm{C}_{5}$ triazole), 129, 127, 116, 163, $56\left(\mathrm{C}_{1}\right.$, and $\mathrm{C}_{2}, \mathrm{C}_{6}$, and $\mathrm{C}_{3}, \mathrm{C}_{5}$, and $\mathrm{C}_{4}$, and $\mathrm{C}_{4}$ 'a respectively), $178(\mathrm{C}$ of $\mathrm{NHCO}), 59\left(\mathrm{C}\right.$ of $\left.\mathrm{CH}_{2} \mathrm{~N}\right), 57$, 55 and $60\left(\mathrm{C}_{\mathrm{a}}, \mathrm{C}_{\mathrm{e}}\right.$ and $\mathrm{C}_{\mathrm{b}}, \mathrm{C}_{\mathrm{d}}$ and $\mathrm{C}_{\mathrm{f}}$ of piperazine respectively), 62 ( $\mathrm{C}_{\mathrm{g}}$ of 1-benzyl piperazine), 136,

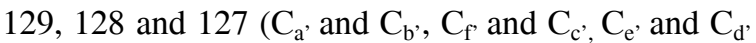
of 1-benzyl-piperazine). FABMS m/z: $438(\mathrm{M}+1)$.

\section{Antimicrobial activity}

Antibacterial studies. The newly synthesized compounds were screened for their antibacterial activity against Escherichia coli (ATCC-8793), Staphylococcus aureus (ATCC-25923),
Pseudomonas aeruginosa (ATCC-27853) and Bacillus subtilis (ATCC-6633) [recultured] bacterial strains by disc diffusion method ${ }^{23}$ at 70,50 and 30 $\mu \mathrm{g} / \mathrm{ml}$ concentrations, respectively. All the bacterial strains were procured from (Hi-media) ATCC, USA. A standard inoculum $\left(1-2 \times 10^{7} \quad\right.$ c.f.u $/ \mathrm{ml} \quad 0.5$ McFarland standards) was introduced onto the surface of sterile agar plates and a sterile glass spreader was used for even distribution of the inoculum. The discs measuring $6 \mathrm{~mm}$ in diameter were prepared from Whatman no. 1 filter paper and sterilized by dry heat at $140^{\circ} \mathrm{C}$ for an hour. The sterile discs previously soaked in a known concentration of the test compounds were placed in nutrient agar medium. Solvent and growth controls were kept. The plates were inverted and incubated for $24 \mathrm{~h}$ at $37^{\circ} \mathrm{C}$. Vancomycin and Amikacin were used as a standard drug. The inhibition zones were measured and compared with the controls.

Antifungal studies. Similarly, the newly prepared compounds were screened for their antifungal activity against Candida albicans (MTCC227), Aspergillus niger (MTCC-3323) and Fusarium oxysporum (MTCC-2087) by paper disc method. All the fungal strains were procured from Institute of Microbial Technology, Chandigarh. For antifungal screening against $A$. niger, czapek yeast extract agar was used. Malt yeast agar $(\approx \mathrm{pH} 7.0)$ was employed as culture media against $C$. albicans and Potato sucrose agar was used as culture medium against F.oxysporum ${ }^{24}$.

\section{RESULTS AND DISCUSSION}

Chemistry. The title compounds $(\mathbf{6 - 1 4 a}, \mathbf{b})$ were synthesized according to Scheme 1. The structure of all the synthesized compounds were elucidated by spectral data. Benzoic acid derivatives were converted into their esters $(\mathbf{1} \mathbf{a}, \mathbf{b})$ using methanol and catalytic amount of sulphuric acid. Esters $(\mathbf{1 a}, \mathbf{b})$ on treatment with hydrazine hydrate yielded corresponding aroyl hydrazides (2-a,b). The IR spectra of $(\mathbf{2} \mathbf{a}, \mathbf{b})$ showed the absence of ester stretching frequency, instead gave a band at around $1650 \mathrm{~cm}^{-1}$ for carbonyl group and showed sharp 
Table 1. Physico-chemical properties of the synthesized compounds.

\begin{tabular}{|c|c|c|c|c|c|c|c|}
\hline $\begin{array}{l}\text { Code } \\
\text { No. }\end{array}$ & $\mathrm{R}$ & $\mathrm{R}^{\prime}$ & Yield (\%) & $\begin{array}{l}\text { M.P. } \\
\left({ }^{\circ} \mathrm{C}\right)\end{array}$ & $\begin{array}{l}\text { Molecular } \\
\text { Formula }\end{array}$ & $\begin{array}{l}\text { Nitrogen estimation } \\
\text { found (calculated) } \\
(\%)\end{array}$ & $\log \mathrm{P}$ \\
\hline $6 a$ & $-\mathrm{CH}_{3}$ & & 65 & 134 & $\mathrm{C}_{16} \mathrm{H}_{21} \mathrm{~N}_{5} \mathrm{SO}$ & 21.09 (21.13) & 3.89 \\
\hline $7 \mathrm{a}$ & $-\mathrm{CH}_{3}$ & & 72 & 126 & $\mathrm{C}_{17} \mathrm{H}_{23} \mathrm{~N}_{5} \mathrm{SO}$ & $20.23(20.27)$ & 4.38 \\
\hline $8 \mathrm{a}$ & $-\mathrm{CH}_{3}$ & & 81 & 105 & $\mathrm{C}_{17} \mathrm{H}_{23} \mathrm{~N}_{5} \mathrm{SO}$ & $20.24(20.27)$ & 4.38 \\
\hline $9 a$ & $-\mathrm{CH}_{3}$ & & 63 & 122 & $\mathrm{C}_{18} \mathrm{H}_{25} \mathrm{~N}_{5} \mathrm{SO}$ & $19.52(19.48)$ & 4.91 \\
\hline $10 \mathrm{a}$ & $-\mathrm{CH}_{3}$ & & 58 & 150 & $\mathrm{C}_{23} \mathrm{H}_{27} \mathrm{~N}_{5} \mathrm{SO}$ & $16.58(16.61)$ & 5.88 \\
\hline $11 \mathrm{a}$ & $-\mathrm{CH}_{3}$ & & 63 & 148 & $\mathrm{C}_{15} \mathrm{H}_{19} \mathrm{~N}_{5} \mathrm{SO}_{2}$ & $21.04(21.01)$ & 2.57 \\
\hline $12 \mathrm{a}$ & $-\mathrm{CH}_{3}$ & & 72 & 176 & $\mathrm{C}_{15} \mathrm{H}_{19} \mathrm{~N}_{5} \mathrm{SO}$ & $22.10(22.06)$ & 3.32 \\
\hline $13 a$ & $-\mathrm{CH}_{3}$ & & 70 & 116 & $\mathrm{C}_{15} \mathrm{H}_{17} \mathrm{~N}_{5} \mathrm{SO}_{2}$ & $21.16(21.13)$ & 3.40 \\
\hline $14 \mathrm{a}$ & $-\mathrm{CH}_{3}$ & & 62 & 140 & $\mathrm{C}_{22} \mathrm{H}_{26} \mathrm{~N}_{6} \mathrm{SO}$ & $19.83(19.89)$ & 4.18 \\
\hline $6 b$ & $-\mathrm{OCH}_{3}$ & & 64 & 201 & $\mathrm{C}_{16} \mathrm{H}_{21} \mathrm{~N}_{5} \mathrm{SO}_{2}$ & $20.13(20.16)$ & 3.59 \\
\hline $7 b$ & $-\mathrm{OCH}_{3}$ & & 41 & 166 & $\mathrm{C}_{17} \mathrm{H}_{23} \mathrm{~N}_{5} \mathrm{SO}_{2}$ & $19.42(19.38)$ & 4.08 \\
\hline $8 \mathrm{~b}$ & $-\mathrm{OCH}_{3}$ & & 67 & 178 & $\mathrm{C}_{17} \mathrm{H}_{23} \mathrm{~N}_{5} \mathrm{SO}_{2}$ & $19.35(19.38)$ & 4.08 \\
\hline $9 b$ & $-\mathrm{OCH}_{3}$ & & 55 & 185 & $\mathrm{C}_{18} \mathrm{H}_{25} \mathrm{~N}_{5} \mathrm{SO}_{2}$ & $18.68(18.65)$ & 4.62 \\
\hline $10 \mathrm{~b}$ & $-\mathrm{OCH}_{3}$ & & 59 & 163 & $\mathrm{C}_{23} \mathrm{H}_{27} \mathrm{~N}_{5} \mathrm{SO}_{2}$ & $13.20(13.23)$ & 5.58 \\
\hline $11 \mathrm{~b}$ & $-\mathrm{OCH}_{3}$ & & 74 & 186 & $\mathrm{C}_{15} \mathrm{H}_{19} \mathrm{~N}_{5} \mathrm{SO}_{3}$ & $20.08(20.04)$ & 2.28 \\
\hline $12 b$ & $-\mathrm{OCH}_{3}$ & & 61 & 196 & $\mathrm{C}_{15} \mathrm{H}_{19} \mathrm{~N}_{5} \mathrm{SO}_{2}$ & $21.04(21.01)$ & 3.03 \\
\hline $13 b$ & $-\mathrm{OCH}_{3}$ & & 65 & 138 & $\mathrm{C}_{15} \mathrm{H}_{17} \mathrm{~N}_{5} \mathrm{SO}_{3}$ & $20.13(20.16)$ & 3.11 \\
\hline $14 \mathrm{~b}$ & $-\mathrm{OCH}_{3}$ & & 55 & 181 & $\mathrm{C}_{22} \mathrm{H}_{26} \mathrm{~N}_{6} \mathrm{SO}_{2}$ & $19.12(19.16)$ & 3.88 \\
\hline
\end{tabular}


Table 2. Antibacterial activity of the synthesized compounds (6-14 a,b).

\begin{tabular}{|c|c|c|c|c|c|c|c|c|c|c|c|c|}
\hline \multirow[t]{2}{*}{ Comp. } & \multicolumn{3}{|c|}{ S. aures } & \multicolumn{3}{|c|}{ B. subtilis } & \multicolumn{3}{|c|}{ P. aeruginosa } & \multicolumn{3}{|c|}{ E.coli } \\
\hline & $\begin{array}{c}70 \\
\mu \mathrm{g} / \mathrm{ml}\end{array}$ & $\begin{array}{c}50 \\
\mu \mathrm{g} / \mathrm{ml}\end{array}$ & $\begin{array}{c}30 \\
\mu \mathrm{g} / \mathrm{ml}\end{array}$ & $\begin{array}{c}70 \\
\mu \mathrm{g} / \mathrm{ml}\end{array}$ & $\begin{array}{c}50 \\
\mu \mathrm{g} / \mathrm{ml}\end{array}$ & $\begin{array}{c}30 \\
\mu \mathrm{g} / \mathrm{ml}\end{array}$ & $\begin{array}{c}70 \\
\mu \mathrm{g} / \mathrm{ml}\end{array}$ & $\begin{array}{c}50 \\
\mu \mathrm{g} / \mathrm{ml}\end{array}$ & $\begin{array}{c}30 \\
\mu \mathrm{g} / \mathrm{ml}\end{array}$ & $\begin{array}{c}70 \\
\mu \mathrm{g} / \mathrm{ml}\end{array}$ & $50 \mu \mathrm{g} / \mathrm{ml}$ & $\begin{array}{c}30 \\
\mu \mathrm{g} / \mathrm{ml}\end{array}$ \\
\hline $6 a$ & $\begin{array}{c}8.26 \\
\pm 0.38\end{array}$ & $\begin{array}{c}7.23 \\
\pm 0.55\end{array}$ & $\begin{array}{c}6.40 \\
\pm 0.46\end{array}$ & $\begin{array}{c}8.13 \\
\pm 0.56\end{array}$ & $\begin{array}{c}7.16 \\
\pm 0.47\end{array}$ & $\begin{array}{c}6.16 \\
\pm 0.70\end{array}$ & $\begin{array}{c}9.20 \\
\pm 0.70\end{array}$ & $\begin{array}{c}8.00 \\
\pm 0.80\end{array}$ & $\begin{array}{c}7.33 \\
\pm 1.14\end{array}$ & $\begin{array}{c}7.33 \\
\pm 0.56\end{array}$ & $\begin{array}{c}6.50 \\
\pm 0.60\end{array}$ & $\begin{array}{c}6.20 \\
\pm 0.66\end{array}$ \\
\hline $7 a$ & $\begin{array}{c}9.13 \\
\pm 0.56\end{array}$ & $\begin{array}{c}8.13 \\
\pm 0.56\end{array}$ & $\begin{array}{c}7.16 \\
\pm 0.70\end{array}$ & $\begin{array}{c}9.20 \\
\pm 0.70\end{array}$ & $\begin{array}{c}8.06 \\
\pm 0.65\end{array}$ & $\begin{array}{c}7.17 \\
\pm 0.56\end{array}$ & $\begin{array}{l}10.12 \\
\pm 0.56\end{array}$ & $\begin{array}{c}9.23 \\
\pm 0.65\end{array}$ & $\begin{array}{c}8.26 \\
\pm 0.68\end{array}$ & $\begin{array}{c}8.23 \\
\pm 0.60\end{array}$ & $\begin{array}{c}7.13 \\
\pm 0.56\end{array}$ & $\begin{array}{c}6.30 \\
\pm 0.46\end{array}$ \\
\hline $8 a$ & $\begin{array}{c}7.03 \\
\pm 0.56\end{array}$ & $\begin{array}{c}6.16 \\
\pm 0.70\end{array}$ & $\begin{array}{c}6.13 \\
\pm 0.56\end{array}$ & $\begin{array}{c}8.10 \\
\pm 0.46\end{array}$ & $\begin{array}{c}7.76 \\
\pm 0.61\end{array}$ & $\begin{array}{c}6.10 \\
\pm 0.10\end{array}$ & $\begin{array}{c}7.16 \\
\pm 0.47\end{array}$ & $\begin{array}{c}6.40 \\
\pm 0.46\end{array}$ & $\begin{array}{c}6.16 \\
\pm 0.47\end{array}$ & $\begin{array}{c}6.46 \\
\pm 0.71\end{array}$ & $\begin{array}{c}6.33 \\
\pm 0.21\end{array}$ & $\begin{array}{c}6.16 \\
\pm 0.70\end{array}$ \\
\hline $9 \mathrm{a}$ & $\begin{array}{c}8.03 \\
\pm 0.56\end{array}$ & $\begin{array}{c}7.10 \\
\pm 0.70\end{array}$ & $\begin{array}{c}6.16 \\
\pm 0.80\end{array}$ & $\begin{array}{c}7.03 \\
\pm 0.56\end{array}$ & $\begin{array}{c}6.16 \\
\pm 0.70\end{array}$ & $\begin{array}{c}6.13 \\
\pm 0.56\end{array}$ & $\begin{array}{c}8.96 \\
\pm 0.55\end{array}$ & $\begin{array}{c}7.10 \\
\pm 0.56\end{array}$ & $\begin{array}{c}6.63 \\
\pm 0.56\end{array}$ & $\begin{array}{c}8.40 \\
\pm 0.56\end{array}$ & $\begin{array}{c}7.10 \\
\pm 0.70\end{array}$ & $\begin{array}{c}6.46 \\
\pm 0.71\end{array}$ \\
\hline $10 \mathrm{a}$ & $\begin{array}{l}10.23 \\
\pm 0.70\end{array}$ & $\begin{array}{c}9.23 \\
\pm 0.55\end{array}$ & $\begin{array}{c}8.40 \\
\pm 0.46\end{array}$ & $\begin{array}{c}8.10 \\
\pm 0.75\end{array}$ & $\begin{array}{c}7.06 \\
\pm 0.65\end{array}$ & $\begin{array}{c}6.13 \\
\pm 0.56\end{array}$ & $\begin{array}{c}7.10 \\
\pm 0.46\end{array}$ & $\begin{array}{c}6.76 \\
\pm 0.61\end{array}$ & $\begin{array}{c}6.17 \\
\pm 0.56\end{array}$ & $\begin{array}{c}8.20 \\
\pm 0.66\end{array}$ & $\begin{array}{c}7.23 \\
\pm 0.70\end{array}$ & $\begin{array}{c}6.83 \\
\pm 0.56\end{array}$ \\
\hline $11 \mathrm{a}$ & $\begin{array}{l}12.26 \\
\pm 0.38\end{array}$ & $\begin{array}{l}10.23 \\
\pm 0.55\end{array}$ & $\begin{array}{c}9.40 \\
\pm 0.46\end{array}$ & $\begin{array}{l}11.53 \\
\pm 0.55\end{array}$ & $\begin{array}{l}10.27 \\
\pm 0.56\end{array}$ & $\begin{array}{c}9.10 \\
\pm 0.76\end{array}$ & $\begin{array}{l}13.13 \\
\pm 0.56\end{array}$ & $\begin{array}{l}12.20 \\
\pm 0.70\end{array}$ & $\begin{array}{l}11.16 \\
\pm 0.47\end{array}$ & $\begin{array}{l}12.20 \\
\pm 0.56\end{array}$ & $\begin{array}{l}11.50 \\
\pm 0.56\end{array}$ & $\begin{array}{l}10.06 \\
\pm 0.56\end{array}$ \\
\hline $12 \mathrm{a}$ & $\begin{array}{c}6.20 \\
\pm 0.46\end{array}$ & $\begin{array}{c}6.17 \\
\pm 0.56\end{array}$ & $\begin{array}{c}6.10 \\
\pm 0.10\end{array}$ & $\begin{array}{c}6.33 \\
\pm 0.21\end{array}$ & $\begin{array}{c}6.17 \\
\pm 0.56\end{array}$ & $\begin{array}{c}6.10 \\
\pm 0.10\end{array}$ & $\begin{array}{c}6.76 \\
\pm 0.61\end{array}$ & $\begin{array}{c}6.33 \\
\pm 0.21\end{array}$ & $\begin{array}{c}6.16 \\
\pm 0.70\end{array}$ & $\begin{array}{c}6.40 \\
\pm 0.46\end{array}$ & $\begin{array}{c}6.33 \\
\pm 0.21\end{array}$ & $\begin{array}{c}6.17 \\
\pm 0.56\end{array}$ \\
\hline $13 \mathrm{a}$ & $\begin{array}{c}7.13 \\
\pm 0.65\end{array}$ & $\begin{array}{c}6.20 \\
\pm 0.53\end{array}$ & $\begin{array}{c}6.10 \\
\pm 0.10\end{array}$ & $\begin{array}{c}7.40 \\
\pm 0.30\end{array}$ & $\begin{array}{c}6.40 \\
\pm 0.46\end{array}$ & $\begin{array}{c}6.13 \\
\pm 0.56\end{array}$ & $\begin{array}{c}8.06 \\
\pm 0.65\end{array}$ & $\begin{array}{c}7.80 \\
\pm 0.56\end{array}$ & $\begin{array}{c}6.10 \\
\pm 0.56\end{array}$ & $\begin{array}{c}7.03 \\
\pm 0.65\end{array}$ & $\begin{array}{c}6.13 \\
\pm 0.56\end{array}$ & $\begin{array}{c}6.10 \\
\pm 0.56\end{array}$ \\
\hline $14 \mathrm{a}$ & $\begin{array}{c}8.16 \\
\pm 0.56\end{array}$ & $\begin{array}{c}7.10 \\
\pm 0.75\end{array}$ & $\begin{array}{c}6.46 \\
\pm 0.71\end{array}$ & $\begin{array}{c}7.10 \\
\pm 0.56\end{array}$ & $\begin{array}{c}6.20 \\
\pm 0.70\end{array}$ & $\begin{array}{c}6.10 \\
\pm 0.10\end{array}$ & $\begin{array}{c}8.20 \\
\pm 0.56\end{array}$ & $\begin{array}{c}7.13 \\
\pm 0.70\end{array}$ & $\begin{array}{c}6.20 \\
\pm 0.70\end{array}$ & $\begin{array}{c}9.70 \\
\pm 0.45\end{array}$ & $\begin{array}{c}8.23 \\
\pm 0.61\end{array}$ & $\begin{array}{c}7.23 \\
\pm 1.04\end{array}$ \\
\hline $6 b$ & $\begin{array}{l}10.30 \\
\pm 0.56\end{array}$ & $\begin{array}{c}9.23 \\
\pm 0.70\end{array}$ & $\begin{array}{c}8.20 \\
\pm 0.56\end{array}$ & $\begin{array}{c}9.20 \\
\pm 0.56\end{array}$ & $\begin{array}{c}8.30 \\
\pm 0.65\end{array}$ & $\begin{array}{c}7.26 \\
\pm 0.90\end{array}$ & $\begin{array}{l}11.23 \\
\pm 0.55\end{array}$ & $\begin{array}{c}9.20 \\
\pm 0.56\end{array}$ & $\begin{array}{c}8.13 \\
\pm 0.65\end{array}$ & $\begin{array}{l}10.26 \\
\pm 0.56\end{array}$ & $\begin{array}{c}9.16 \\
\pm 0.56\end{array}$ & $\begin{array}{c}8.26 \\
\pm 0.65\end{array}$ \\
\hline $7 b$ & $\begin{array}{c}9.16 \\
\pm 0.60\end{array}$ & $\begin{array}{c}8.23 \\
\pm 0.65\end{array}$ & $\begin{array}{c}7.65 \\
\pm 0.55\end{array}$ & $\begin{array}{l}10.16 \\
\pm 0.65\end{array}$ & $\begin{array}{c}9.26 \\
\pm 1.06\end{array}$ & $\begin{array}{c}8.14 \\
\pm 0.56\end{array}$ & $\begin{array}{c}8.13 \\
\pm 0.56\end{array}$ & $\begin{array}{c}7.12 \\
\pm 0.56\end{array}$ & $\begin{array}{c}6.26 \\
\pm 0.92\end{array}$ & $\begin{array}{c}8.20 \\
\pm 0.56\end{array}$ & $\begin{array}{c}7.26 \\
\pm 0.60\end{array}$ & $\begin{array}{c}6.23 \\
\pm 0.60\end{array}$ \\
\hline $8 b$ & $\begin{array}{c}8.03 \\
\pm 0.56\end{array}$ & $\begin{array}{c}7.06 \\
\pm 0.65\end{array}$ & $\begin{array}{c}6.20 \\
\pm 0.70\end{array}$ & $\begin{array}{c}7.03 \\
\pm 0.75\end{array}$ & $\begin{array}{c}6.40 \\
\pm 0.30\end{array}$ & $\begin{array}{c}6.10 \\
\pm 0.10\end{array}$ & $\begin{array}{c}8.03 \\
\pm 0.75\end{array}$ & $\begin{array}{c}7.10 \\
\pm 0.76\end{array}$ & $\begin{array}{c}6.20 \\
\pm 1.10\end{array}$ & $\begin{array}{c}9.03 \\
\pm 0.56\end{array}$ & $\begin{array}{c}8.16 \\
\pm 0.56\end{array}$ & $\begin{array}{c}7.30 \\
\pm 0.56\end{array}$ \\
\hline $9 b$ & $\begin{array}{l}11.13 \\
\pm 0.56\end{array}$ & $\begin{array}{l}10.16 \\
\pm 0.75\end{array}$ & $\begin{array}{c}9.03 \\
\pm 0.56\end{array}$ & $\begin{array}{c}7.06 \\
\pm 0.56\end{array}$ & $\begin{array}{c}6.26 \\
\pm 0.75\end{array}$ & $\begin{array}{c}6.10 \\
\pm 0.75\end{array}$ & $\begin{array}{l}10.56 \\
\pm 0.56\end{array}$ & $\begin{array}{c}9.16 \\
\pm 0.75\end{array}$ & $\begin{array}{c}8.63 \\
\pm 0.56\end{array}$ & $\begin{array}{c}9.16 \\
\pm 0.56\end{array}$ & $\begin{array}{c}8.20 \\
\pm 0.82\end{array}$ & $\begin{array}{c}7.30 \\
\pm 0.46\end{array}$ \\
\hline $10 \mathrm{~b}$ & $\begin{array}{c}8.23 \\
\pm 0.56\end{array}$ & $\begin{array}{c}7.16 \\
\pm 0.56\end{array}$ & $\begin{array}{c}6.33 \\
\pm 0.55\end{array}$ & $\begin{array}{c}8.23 \\
\pm 0.60\end{array}$ & $\begin{array}{c}7.43 \\
\pm 0.70\end{array}$ & $\begin{array}{c}6.16 \\
\pm 0.47\end{array}$ & $\begin{array}{c}9.23 \\
\pm 0.56\end{array}$ & $\begin{array}{c}8.13 \\
\pm 0.56\end{array}$ & $\begin{array}{c}7.33 \\
\pm 0.56\end{array}$ & $\begin{array}{l}10.16 \\
\pm 0.56\end{array}$ & $\begin{array}{c}9.13 \\
\pm 0.56\end{array}$ & $\begin{array}{c}8.80 \\
\pm 0.56\end{array}$ \\
\hline $11 b$ & $\begin{array}{l}15.06 \\
\pm 0.56\end{array}$ & $\begin{array}{l}12.23 \\
\pm 0.56\end{array}$ & $\begin{array}{l}11.10 \\
\pm 0.56\end{array}$ & $\begin{array}{l}13.96 \\
\pm 0.56\end{array}$ & $\begin{array}{l}12.03 \\
\pm 0.80\end{array}$ & $\begin{array}{l}11.16 \\
\pm 0.80\end{array}$ & $\begin{array}{l}16.13 \\
\pm 0.70\end{array}$ & $\begin{array}{l}14.03 \\
\pm 0.56\end{array}$ & $\begin{array}{l}13.16 \\
\pm 0.75\end{array}$ & $\begin{array}{l}15.23 \\
\pm 0.65\end{array}$ & $\begin{array}{l}13.17 \\
\pm 0.75\end{array}$ & $\begin{array}{l}12.16 \\
\pm 0.80\end{array}$ \\
\hline $12 \mathrm{~b}$ & $\begin{array}{c}9.10 \\
\pm 0.70\end{array}$ & $\begin{array}{c}8.23 \\
\pm 0.56\end{array}$ & $\begin{array}{c}7.10 \\
\pm 0.56\end{array}$ & $\begin{array}{c}7.43 \\
\pm 0.70\end{array}$ & $\begin{array}{c}6.33 \\
\pm 0.21\end{array}$ & $\begin{array}{c}6.13 \\
\pm 0.65\end{array}$ & $\begin{array}{c}8.10 \\
\pm 0.56\end{array}$ & $\begin{array}{c}7.26 \\
\pm 0.56\end{array}$ & $\begin{array}{c}6.56 \\
\pm 0.56\end{array}$ & $\begin{array}{c}7.03 \\
\pm 0.56\end{array}$ & $\begin{array}{c}6.26 \\
\pm 0.65\end{array}$ & $\begin{array}{c}6.06 \\
\pm 0.56\end{array}$ \\
\hline $13 b$ & $\begin{array}{c}6.33 \\
\pm 0.21\end{array}$ & $\begin{array}{c}6.23 \\
\pm 0.21\end{array}$ & $\begin{array}{c}6.10 \\
\pm 0.10\end{array}$ & $\begin{array}{c}7.16 \\
\pm 0.75\end{array}$ & $\begin{array}{c}6.36 \\
\pm 0.64\end{array}$ & $\begin{array}{c}6.13 \\
\pm 0.65\end{array}$ & $\begin{array}{l}10.16 \\
\pm 0.65\end{array}$ & $\begin{array}{c}9.10 \\
\pm 0.80\end{array}$ & $\begin{array}{c}8.20 \\
\pm 0.65\end{array}$ & $\begin{array}{c}8.13 \\
\pm 0.86\end{array}$ & $\begin{array}{c}7.03 \\
\pm 0.56\end{array}$ & $\begin{array}{c}6.20 \\
\pm 0.75\end{array}$ \\
\hline $14 \mathrm{~b}$ & $\begin{array}{l}10.06 \\
\pm 0.70\end{array}$ & $\begin{array}{c}9.10 \\
\pm 0.80\end{array}$ & $\begin{array}{c}8.20 \\
\pm 0.75\end{array}$ & $\begin{array}{c}8.06 \\
\pm 0.56\end{array}$ & $\begin{array}{c}7.17 \\
\pm 0.56\end{array}$ & $\begin{array}{c}6.20 \\
\pm 0.56\end{array}$ & $\begin{array}{l}10.23 \\
\pm 0.70\end{array}$ & $\begin{array}{c}9.10 \\
\pm 0.56\end{array}$ & $\begin{array}{c}8.23 \\
\pm 0.56\end{array}$ & $\begin{array}{l}14.80 \\
\pm 0.56\end{array}$ & $\begin{array}{l}12.20 \\
\pm 0.65\end{array}$ & $\begin{array}{r}11.20 \\
\pm 0.70\end{array}$ \\
\hline Vanc & mycin & & $\begin{array}{l}18.20 \\
\pm 0.30\end{array}$ & & & $\begin{array}{l}17.43 \\
\pm 0.35\end{array}$ & & & & & & \\
\hline Am & racin & & & & & & & & $\begin{array}{l}22.10 \\
\pm 0.36\end{array}$ & & & $\begin{array}{l}21.27 \\
\pm 0.56\end{array}$ \\
\hline
\end{tabular}

bands in the region of $3300-3430 \mathrm{~cm}^{-1}$ due to $-\mathrm{NHNH}_{2}$ group. Aroyl hydrazides $(\mathbf{2 a}, \mathbf{b})$ on reacting with alcoholic potassium hydroxide and carbon disulfide yielded corresponding potassium 3-aroyl dithiocarbazate $(\mathbf{3 a}, \mathbf{b})$. The IR spectra of $\mathbf{3 a}, \mathbf{b}$ showed the presence of $\mathrm{C}=\mathrm{S}$ stretching at around $1129 \mathrm{~cm}^{-1}$ and $\mathrm{C}=\mathrm{O}$ stretching of amide at around $1667 \mathrm{~cm}^{-1}$. Potassium 3-aroyl dithiocarbazate $(\mathbf{3 a}, \mathbf{b})$ on cyclization reaction with ethanol and hydrazine hydrate yielded 4-amino-3-mercapto-5-substitutedphenyl 1,2,4-triazole (4a,b). 1,2,4-Triazole showed
$\mathrm{C}-\mathrm{N}$ stretching at around $1380 \mathrm{~cm}^{-1}$ and $\mathrm{C}=\mathrm{N}$ stretching at around $1530 \mathrm{~cm}^{-1}$. Free thiol group which was attached to 1,2,4-triazole showed stretching band at around $2520-2580 \mathrm{~cm}^{-1}$ and 3200$3300 \mathrm{~cm}^{-1}$ due to amino group. Signal at around $\delta \mathbf{e}$ 143 ppm confirmed the C-3 and C-5 carbon of 1,2,4triazole ring. 4-amino-3-mercapto-5-substitutedphenyl 1,2,4-triazole $(\mathbf{4 a}, \mathbf{b})$ on treatment with chloroacetyl chloride in dioxane yielded 4-(chloroacetyl amino)-3-mercapto-5-(4-substituted) phenyl1,2,4-triazole (5a,b). The IR spectra of 5a,b showed 
the disappearance of 3200-3300 stretching of amino group. Signals at around $\delta \mathrm{e} 165 \mathrm{ppm}$ and $53 \mathrm{ppm}$ confirmed the $-\mathrm{NHCO}$ and $\mathrm{CH}_{2}$ group. 4-(Chloroacetyl amino)-3-mercapto-5-(4-substituted) phenyl1,2,4-triazole $\mathbf{( 5 a , b )}$ on treatment with different secondary amines in triethyl amine yielded title compounds (6-14a,b) (Scheme 1). IR spectra showed $\mathrm{C}-\mathrm{N}$ and $\mathrm{C}=\mathrm{N}$ stretching frequencies of triazole at around $1350 \mathrm{~cm}^{-1}$ and $1540 \mathrm{~cm}^{-1}$, respectively.
Aromatic C-H stretching of phenyl ring appeared around 3000-3100 $\mathrm{cm}^{-1}$. Further ${ }^{13} \mathrm{C}-\mathrm{NMR}$ spectra, which showed $\mathrm{C}_{3}$ and $\mathrm{C}_{5}$ signal of 1,2,4-triazole at around $\delta e 145 \mathrm{ppm}$. Carbonyl carbon and methylene carbon of $-\mathrm{NHCOCH}_{2} \mathrm{~N}<$ were seen at $\delta$ e $173 \mathrm{ppm}$ and $56 \mathrm{ppm}$, respectively and ${ }^{1} \mathrm{H}$ NMR spectra, showed singlet of thiol group between 8.67-9.92 ppm, singlet of -CONH between 9.87-10.16 ppm.

Table 3. Antifungal activity of the synthesized compounds (6-14 a,b).

\begin{tabular}{|c|c|c|c|c|c|c|c|c|c|}
\hline \multirow[t]{2}{*}{ Comp. } & \multicolumn{3}{|c|}{ C. albicans } & \multicolumn{3}{|c|}{ A. niger } & \multicolumn{3}{|c|}{ F. oxysporum } \\
\hline & $\begin{array}{c}70 \\
\mu \mathrm{g} / \mathrm{ml} \\
\end{array}$ & $\begin{array}{c}50 \\
\mu \mathrm{g} / \mathrm{ml} \\
\end{array}$ & $\begin{array}{c}30 \\
\mu \mathrm{g} / \mathrm{ml} \\
\end{array}$ & $\begin{array}{c}70 \\
\mu \mathrm{g} / \mathrm{ml} \\
\end{array}$ & $\begin{array}{c}50 \\
\mu \mathrm{g} / \mathrm{ml} \\
\end{array}$ & $\begin{array}{c}30 \\
\mu \mathrm{g} / \mathrm{ml} \\
\end{array}$ & $\begin{array}{c}70 \\
\mu \mathrm{g} / \mathrm{ml} \\
\end{array}$ & $\begin{array}{c}50 \\
\mu \mathrm{g} / \mathrm{ml} \\
\end{array}$ & $\begin{array}{c}30 \\
\mu \mathrm{g} / \mathrm{ml}\end{array}$ \\
\hline $6 a$ & $\begin{array}{c}7.13 \\
\pm 0.75\end{array}$ & $\begin{array}{c}6.40 \\
\pm 0.46\end{array}$ & $\begin{array}{c}6.26 \\
\pm 0.60\end{array}$ & $\begin{array}{l}10.13 \\
\pm 0.65\end{array}$ & $\begin{array}{c}9.16 \\
\pm 0.75\end{array}$ & $\begin{array}{c}7.26 \\
\pm 0.65\end{array}$ & $\begin{array}{c}9.03 \\
\pm 1.05\end{array}$ & $\begin{array}{c}8.30 \\
\pm 0.70\end{array}$ & $\begin{array}{c}7.23 \\
\pm 0.65\end{array}$ \\
\hline $7 \mathrm{a}$ & $\begin{array}{c}9.16 \\
\pm 0.55\end{array}$ & $\begin{array}{c}8.20 \\
\pm 0.76\end{array}$ & $\begin{array}{c}7.26 \\
\pm 0.65\end{array}$ & $\begin{array}{l}11.16 \\
\pm 0.70\end{array}$ & $\begin{array}{l}10.10 \\
\pm 0.56\end{array}$ & $\begin{array}{c}9.13 \\
\pm 0.70\end{array}$ & $\begin{array}{l}10.20 \\
\pm 0.65\end{array}$ & $\begin{array}{c}9.16 \\
\pm 0.71\end{array}$ & $\begin{array}{c}8.16 \\
\pm 0.55\end{array}$ \\
\hline $8 a$ & $\begin{array}{c}7.13 \\
\pm 0.71\end{array}$ & $\begin{array}{c}6.17 \\
\pm 0.65\end{array}$ & $\begin{array}{c}6.06 \\
\pm 0.65\end{array}$ & $\begin{array}{c}7.03 \\
\pm 0.55\end{array}$ & $\begin{array}{c}6.33 \\
\pm 0.21\end{array}$ & $\begin{array}{c}6.16 \\
\pm 0.70\end{array}$ & $\begin{array}{c}9.06 \\
\pm 0.60\end{array}$ & $\begin{array}{c}8.16 \\
\pm 0.75\end{array}$ & $\begin{array}{c}7.26 \\
\pm 0.65\end{array}$ \\
\hline $9 \mathrm{a}$ & $\begin{array}{c}8.21 \\
\pm 0.66\end{array}$ & $\begin{array}{c}7.16 \\
\pm 0.75\end{array}$ & $\begin{array}{c}6.23 \\
\pm 0.70\end{array}$ & $\begin{array}{c}8.23 \\
\pm 0.55\end{array}$ & $\begin{array}{c}7.26 \\
\pm 0.81\end{array}$ & $\begin{array}{c}6.17 \\
\pm 0.65\end{array}$ & $\begin{array}{c}7.06 \\
\pm 0.65\end{array}$ & $\begin{array}{c}6.26 \\
\pm 1.20\end{array}$ & $\begin{array}{c}6.06 \\
\pm 1.30\end{array}$ \\
\hline $10 \mathrm{a}$ & $\begin{array}{c}7.13 \\
\pm 0.65\end{array}$ & $\begin{array}{c}6.33 \\
\pm 0.55\end{array}$ & $\begin{array}{c}6.26 \\
\pm 0.65\end{array}$ & $\begin{array}{c}8.16 \\
\pm 0.60\end{array}$ & $\begin{array}{c}7.16 \\
\pm 0.75\end{array}$ & $\begin{array}{c}6.26 \\
\pm 0.60\end{array}$ & $\begin{array}{c}7.13 \\
\pm 0.65\end{array}$ & $\begin{array}{c}6.33 \\
\pm 0.55\end{array}$ & $\begin{array}{c}6.16 \\
\pm 0.75\end{array}$ \\
\hline $11 \mathrm{a}$ & $\begin{array}{l}11.17 \\
\pm 0.55\end{array}$ & $\begin{array}{l}10.00 \\
\pm 0.98\end{array}$ & $\begin{array}{c}8.27 \\
\pm 0.65\end{array}$ & $\begin{array}{l}15.03 \\
\pm 0.86\end{array}$ & $\begin{array}{l}14.20 \\
\pm 0.76\end{array}$ & $\begin{array}{l}12.16 \\
\pm 0.47\end{array}$ & $\begin{array}{c}8.21 \\
\pm 0.65\end{array}$ & $\begin{array}{c}7.17 \\
\pm 0.65\end{array}$ & $\begin{array}{c}6.23 \\
\pm 0.65\end{array}$ \\
\hline $12 \mathrm{a}$ & $\begin{array}{c}7.10 \\
\pm 0.56\end{array}$ & $\begin{array}{c}6.20 \\
\pm 0.72\end{array}$ & $\begin{array}{c}6.13 \\
\pm 0.65\end{array}$ & $\begin{array}{c}7.20 \\
\pm 0.65\end{array}$ & $\begin{array}{c}6.16 \\
\pm 0.75\end{array}$ & $\begin{array}{c}6.10 \\
\pm 0.10\end{array}$ & $\begin{array}{c}7.76 \\
\pm 0.61\end{array}$ & $\begin{array}{c}6.40 \\
\pm 0.46\end{array}$ & $\begin{array}{c}6.26 \\
\pm 0.60\end{array}$ \\
\hline $13 a$ & $\begin{array}{c}6.33 \\
\pm 0.21\end{array}$ & $\begin{array}{c}6.23 \\
\pm 0.21\end{array}$ & $\begin{array}{c}6.03 \\
\pm 0.60\end{array}$ & $\begin{array}{c}7.23 \\
\pm 0.70\end{array}$ & $\begin{array}{c}7.17 \\
\pm 0.60\end{array}$ & $\begin{array}{c}6.67 \\
\pm 0.45\end{array}$ & $\begin{array}{c}6.33 \\
\pm 0.21\end{array}$ & $\begin{array}{c}6.17 \\
\pm 0.65\end{array}$ & $\begin{array}{c}6.03 \\
\pm 0.65\end{array}$ \\
\hline $14 \mathrm{a}$ & $\begin{array}{c}8.26 \\
\pm 0.65\end{array}$ & $\begin{array}{c}7.46 \\
\pm 0.71\end{array}$ & $\begin{array}{c}6.23 \\
\pm 0.65\end{array}$ & $\begin{array}{c}9.13 \\
\pm 0.55\end{array}$ & $\begin{array}{c}8.23 \\
\pm 0.60\end{array}$ & $\begin{array}{c}7.13 \\
\pm 0.65\end{array}$ & $\begin{array}{c}8.97 \\
\pm 0.65\end{array}$ & $\begin{array}{c}7.26 \\
\pm 0.75\end{array}$ & $\begin{array}{c}6.03 \\
\pm 1.36\end{array}$ \\
\hline $6 \mathrm{~b}$ & $\begin{array}{l}11.03 \\
\pm 0.55\end{array}$ & $\begin{array}{c}10.13 \\
\pm 0.70\end{array}$ & $\begin{array}{c}9.20 \\
\pm 0.75\end{array}$ & $\begin{array}{l}12.20 \\
\pm 0.56\end{array}$ & $\begin{array}{l}11.20 \\
\pm 0.70\end{array}$ & $\begin{array}{l}10.26 \\
\pm 0.65\end{array}$ & $\begin{array}{l}10.37 \\
\pm 0.55\end{array}$ & $\begin{array}{c}9.10 \\
\pm 0.75\end{array}$ & $\begin{array}{r}8.06 \\
\pm 0.55\end{array}$ \\
\hline $7 \mathrm{~b}$ & $\begin{array}{l}12.03 \\
\pm 0.55\end{array}$ & $\begin{array}{l}11.26 \\
\pm 0.65\end{array}$ & $\begin{array}{c}10.13 \\
\pm 0.70\end{array}$ & $\begin{array}{l}14.10 \\
\pm 0.65\end{array}$ & $\begin{array}{r}12.93 \\
\pm 1.22\end{array}$ & $\begin{array}{l}12.13 \\
\pm 0.65\end{array}$ & $\begin{array}{l}11.10 \\
\pm 0.70\end{array}$ & $\begin{array}{l}10.23 \\
\pm 0.65\end{array}$ & $\begin{array}{c}9.23 \\
\pm 0.65\end{array}$ \\
\hline $8 b$ & $\begin{array}{l}11.10 \\
\pm 0.65\end{array}$ & $\begin{array}{l}10.13 \\
\pm 0.75\end{array}$ & $\begin{array}{c}9.16 \\
\pm 0.89\end{array}$ & $\begin{array}{l}11.21 \\
\pm 0.60\end{array}$ & $\begin{array}{l}10.20 \\
\pm 0.75\end{array}$ & $\begin{array}{c}9.13 \\
\pm 0.70\end{array}$ & $\begin{array}{c}9.06 \\
\pm 0.65\end{array}$ & $\begin{array}{c}8.16 \\
\pm 0.81\end{array}$ & $\begin{array}{c}7.03 \\
\pm 0.65\end{array}$ \\
\hline $9 \mathrm{~b}$ & $\begin{array}{l}13.21 \\
\pm 0.56\end{array}$ & $\begin{array}{l}12.13 \\
\pm 0.65\end{array}$ & $\begin{array}{l}11.06 \\
\pm 0.65\end{array}$ & $\begin{array}{c}9.13 \\
\pm 0.75\end{array}$ & $\begin{array}{c}8.00 \\
\pm 0.70\end{array}$ & $\begin{array}{c}7.03 \\
\pm 0.70\end{array}$ & $\begin{array}{c}7.06 \\
\pm 0.60\end{array}$ & $\begin{array}{c}6.26 \\
\pm 0.65\end{array}$ & $\begin{array}{c}6.16 \\
\pm 0.70\end{array}$ \\
\hline $10 \mathrm{~b}$ & $\begin{array}{c}8.23 \\
\pm 0.65\end{array}$ & $\begin{array}{c}7.13 \\
\pm 0.65\end{array}$ & $\begin{array}{c}6.43 \\
\pm 0.35\end{array}$ & $\begin{array}{l}10.13 \\
\pm 0.65\end{array}$ & $\begin{array}{c}9.03 \\
\pm 0.55\end{array}$ & $\begin{array}{c}8.13 \\
\pm 0.70\end{array}$ & $\begin{array}{c}8.16 \\
\pm 0.65\end{array}$ & $\begin{array}{c}7.20 \\
\pm 0.66\end{array}$ & $\begin{array}{c}6.20 \\
\pm 0.66\end{array}$ \\
\hline $11 \mathrm{~b}$ & $\begin{array}{l}17.13 \\
\pm 0.55\end{array}$ & $\begin{array}{l}16.17 \\
\pm 0.65\end{array}$ & $\begin{array}{l}14.27 \\
\pm 0.65\end{array}$ & $\begin{array}{l}18.03 \\
\pm 0.70\end{array}$ & $\begin{array}{l}16.20 \\
\pm 0.92\end{array}$ & $\begin{array}{l}14.26 \\
\pm 0.45\end{array}$ & $\begin{array}{l}10.13 \\
\pm 0.65\end{array}$ & $\begin{array}{c}9.30 \\
\pm 0.66\end{array}$ & $\begin{array}{c}8.16 \\
\pm 0.65\end{array}$ \\
\hline $12 b$ & $\begin{array}{c}7.80 \\
\pm 0.66\end{array}$ & $\begin{array}{c}6.36 \\
\pm 0.64\end{array}$ & $\begin{array}{c}6.23 \\
\pm 0.70\end{array}$ & $\begin{array}{c}9.16 \\
\pm 0.65\end{array}$ & $\begin{array}{c}8.13 \\
\pm 0.61\end{array}$ & $\begin{array}{c}7.23 \\
\pm 0.70\end{array}$ & $\begin{array}{c}8.03 \\
\pm 0.80\end{array}$ & $\begin{array}{c}7.13 \\
\pm 0.75\end{array}$ & $\begin{array}{c}6.23 \\
\pm 0.65\end{array}$ \\
\hline $13 b$ & $\begin{array}{c}6.76 \\
\pm 0.61\end{array}$ & $\begin{array}{c}6.33 \\
\pm 0.21\end{array}$ & $\begin{array}{c}6.16 \\
\pm 0.70\end{array}$ & $\begin{array}{c}7.10 \\
\pm 0.66\end{array}$ & $\begin{array}{c}6.20 \\
\pm 0.70\end{array}$ & $\begin{array}{c}6.06 \\
\pm 0.70\end{array}$ & $\begin{array}{c}7.10 \\
\pm 0.66\end{array}$ & $\begin{array}{c}6.76 \\
\pm 0.61\end{array}$ & $\begin{array}{c}6.20 \\
\pm 0.70\end{array}$ \\
\hline $14 \mathrm{~b}$ & $\begin{array}{l}10.13 \\
\pm 0.60\end{array}$ & $\begin{array}{c}9.06 \\
\pm 0.65\end{array}$ & $\begin{array}{c}8.23 \\
\pm 0.70\end{array}$ & $\begin{array}{l}11.86 \\
\pm 0.55\end{array}$ & $\begin{array}{l}10.13 \\
\pm 0.80\end{array}$ & $\begin{array}{c}8.96 \\
\pm 0.55\end{array}$ & $\begin{array}{c}6.36 \\
\pm 0.64\end{array}$ & $\begin{array}{c}6.23 \\
\pm 0.70\end{array}$ & $\begin{array}{c}6.10 \\
\pm 0.10\end{array}$ \\
\hline $\begin{array}{c}\text { Clotrim } \\
\text { azole }\end{array}$ & & & $\begin{array}{l}21.87 \\
\pm 1.07\end{array}$ & & & $\begin{array}{l}22.56 \\
\pm 0.51\end{array}$ & & & $\begin{array}{l}19.26 \\
\pm 0.56\end{array}$ \\
\hline
\end{tabular}

Antibacterial activity. The two series of 1,2,4triazole derivatives $(\mathbf{6 - 1 4 a}, \mathbf{b})$ were screened for in vitro antibacterial activity against two gram positive (S. aureus ATCC 25923, B. subtilis ATCC 6633) 
and two gram negative (P. aeruginosa ATCC 27853, E. coli ATCC 8793) recultured bacterial strains by disc diffusion method. ${ }^{23}$ Vancomycin was used as standard drug for gram positive strains and amikacin was used as standard drug for gram negative strains. Results showed that compounds 11a, $6 \mathbf{b}, \mathbf{9 b}, \mathbf{1 1 b}$ and 14b exhibited good antibacterial activity against all tested bacterial strains. Compounds 8a, 12a and 13b axhibited lesser degree of activity. These all active synthesized compounds were more effective against $S$. aureus and $P$. aeruginosa bacterial strains than the two others. The zone of inhibition of the tested compounds at different concentrations are shown in Table 2.

Antifungal activity. For in vitro antifungal activity, three fungal species C. albicans, MTCC 227, $A$. niger MTCC-3323 and $F$. oxysporum, MTCC2087 were used and compared with standard drug clotrimazole. Most of the compounds showed good antifungal activity against $A$. niger compare to other two fungal strains. Compounds 7a, 11a, 6b, 7b and 11b exhibited good antifungal activity against all tested fungal strains. Compounds 8a, 12a, 13a and 13b showed lesser degree of activity. The remaining compounds displayed poor activities against the fungal strains as compared to standard drug clotrimazole. The zone of inhibition of the tested compounds at different concentrations are shown in Table 3.

$\log p$ values demonstrated the crucial role of lipophilicity in determining the antimicrobial activity. Increasing ring size of the compound leads to increase in the $\log p$ value, which further influences antimicrobial activity.

\section{CONCLUSION}

Compounds having piperidine, 2-ethyl piperidine, morpholine and benzyl piperazine group at position 4 of $\mathrm{C}_{2}$ acetamido group of the triazole ring system, displayed good antibacterial activity. SAR studies were also suggesting that the majority of the compounds having 4-methoxy phenyl group at position 5 of the triazole ring showed good antibacterial activity. Antifungal screening confirmed that compounds with piperidine, 2-methyl piperidine and morpholine have significant antifungal activity. Structure activity relationship also reveals that majority of the compounds having para-methoxy phenyl group at position 5 of the triazole ring showed good antifungal activity than the methyl group. So, it seems from antimicrobial results that substitution at phenyl position and at $4^{\text {th }}$ position of triazole ring played vital role in increasing antimicrobial activity.

\section{ACKNOWLEDGEMENTS}

The authors are grateful to the Director, Defence Research Development and Establishment, Gwalior for providing necessary facility and also to Dr. Urmila Tuteja, Head, Microbiology Division, DRDE, Gwalior and Head, Dept. of Pharmaceutical Sciences, Dr. H. S. Gour University, Sagar (M.P.).

\section{REFERENCES}

1. Hof, H. 2008. Is there a serious risk of resistance development to azoles among fungi due to the widespread use and long-termapplication of azole antifungals in medicine? Drug Res. Updat. 11, 25-31.

2. Mathew, V., Keshavayya, J., Acharya, V.P.V. and Reddy, B.M. 2006. Heterocyclic system containing bridgehead nitrogen atom: synthesis and pharmacological activities of some substituted 1,2,4-triazolo[3,4-b]-1,3,4-thiadiazoles. Eur. J. Med. Chem. 41, 1048-1058.

3. Turan-Zitouni, G., Kaplancıklı, Z.A., Yıldı, M.T. and Demet Kaya, P.C. 2005. Synthesis and antimicrobial activity of 4phenyl/cyclohexyl-5-(1-phenoxyethyl)-3-[N-(2-thiazolyl) acetamido]thio-4H-1,2,4-triazole derivatives. Eur. J. Med. Chem. 40, 607-613.

4. Upmanyu, N., Kumar, S., Kharya, M.D., Shah, K. and Mishra P. 2011. Synthesis and antimicrobial evaluation of some novel 1,2,4-triazole derivatives. Acta Pol. Pharm. Drug Res. 68, 213-221.

5. El Rayes, S.M. 2010. Convenient synthesis and antimicrobial activity of some novel amino acid coupled triazoles. Molecules. 15, 6759-6772.

6. Ezabadi, I.R., Canoutsis, C., Zoumpoulakis, P., Geronikaki, A., Sokovic, M., Glamocilija, J. and Ćirić, A. 2008. Sulfonamide-1,2,4-triazole derivatives as antifungal and antibacterial agents: synthesis, biological evaluation, lipophilicity, and conformational studies. Bioorg. Med. Chem. 16, 1150-1161.

7. Kaplancıklı, Z.A., Turan-Zitouni, G., Özdemir, A. and Revial, G. 2008. New triazole and triazolothiadiazine derivatives as possible antimicrobial agents. Eur. J. Med. Chem. 43, 155-159. 
8. Serdar, M., Güumrükçüĝlu, N., Karaoĝlu, Ş.A. and Demirbaş, N. 2007. Synthesis of some novel 3,5-diaryl-1,2,4triazole derivatives and investigation of their antimicrobial activities. Turk. J. Chem. 31, 315-326.

9. Shiradkar, M.R., Murahari, K.K., Gangadasu, H.R., Suresh, T., Kalyan, C.A., Panchal, D., Kaur, R., Burange, P., Ghogare, J., Mokale, V. and Raut, M. 2007. Synthesis of new S-derivatives of clubbed triazolyl thiazole as antiMycobacterium tuberculosis agents. Bioorg. Med. Chem. 15, 3997-4008.

10. Shiradkar, M., Kumar, G.V.S., Dasari, V., Tatikonda, S., Akula, K.C. and Shah, R. 2007. Clubbed triazoles: A novel approach to antitubercular drugs. Eur. J. Med. Chem. 42, 807-816.

11. Sztanke, K., Tuzimski, T., Rzymowska, J., Pasternak, K. and Kandefer-Szerszen, M. 2008. Synthesis, determination of the lipophilicity, anticancer and antimicrobial properties of some fused 1,2,4-triazole derivatives. Eur. J. Med. Chem. 43, 404419.

12. Romagnoli, R., Baraldi, P.G., Cruz-Lopez, O., Cara, C.L., Carrion, M.D., Brancale, A., Hamel, E., Chen, L., Bortolozzi, R., Basso, G. and Viola, G. 2010. Synthesis and antitumor activity of 1,5-disubstituted 1,2,4-triazoles as Cis-restricted combretastatin analogues. J. Med. Chem. 53, 4248-4258.

13. Kumar, H., Javed, S.A., Khan, S.A. and Amir, M. 2008. 1,3,4-Oxadiazole/thiadiazole and 1,2,4-triazole derivatives of biphenyl-4-yloxy acetic acid: Synthesis and preliminary evaluation of biological properties. Eur. J. Med. Chem. 43, 2688-2698.

14. Abdel-Megeed, A.M., Abdel-Rahman, H.M., Alkaramany, G.S. and El-Gendy, M.A. 2009. Design, synthesis and molecular modeling study of acylated 1,2,4-triazole-3acetates with potential anti-inflammatory activity. Eur. J. Med. Chem. 44, 117-123.

15. Navidpour, L., Shafaroodi, H., Abdi, K., Amini, M., Ghahremani, M.H., Dehpourd, A.R. and Shafieea, A. 2006. Design, synthesis, and biological evaluation of substituted 3alkylthio-4,5-diaryl-4H-1,2,4-triazoles as selective COX-2 inhibitors. Bioorg. Med. Chem. 14, 2507-2517.
16. Küçükgüzel, I., Küçükgüzel, Ş.G., Rollas, S., Ötük-Sanıs, G., Özdemir, O., Bayrak, I., Altug, T. and Stables, J.P. 2004. Synthesis of some 3-(Arylalkylthio)-4-alkyl/aryl-5-(4aminophenyl)-4H-1,2,4-triazole derivatives and their anticonvulsant activity. IL FARMACO. 59, 893-901.

17. Cui, X.S., Chen, J., Chai, K.Y., Lee, J.S. and Quan, Z.S. 2009. Synthesis and anticonvulsant evaluation of 3substituted-4-(4-hexyloxyphenyl)-4H-1,2,4-triazoles. $\quad$ Med. Chem. Res. 18, 49-58.

18. Akhtar, T., Hameed, S., Khan, K.M., Khan, A. and Choudhary, M.I. 2010. Design, synthesis, and urease inhibition studies of some 1,3,4-oxadiazoles and 1,2,4triazoles derived from mandelic acid. J. Enz. Inhibit. Med. Chem. 25, 572-576.

19. Romine, J.L., Martin, S.W., Gribkoff, V.K., Boissard, C.G., Dworetzky, S.I., Natale, J., Li, Y., Gao, Q., Meanwell, N.A. and Starrett, J.E. 2002. 4,5-Diphenyltriazol-3-ones: Openers of large-conductance $\mathrm{Ca}^{2+}$-activated potassium (Maxi-K) channels. J. Med. Chem. 45, 2942-2952.

20. Zhang, Q., Peng, Y., Wang, X.I., Keenan, S.M., Arora, S. and Welsh, W.J. 2007. Highly potent triazole-based tubulin polymerization inhibitors. J. Med. Chem. 50, 749-754.

21. Moulin, A., Demange, L., Ryan, J., Mousseaux, D., Sanchez, P., Bergé, G., Gagne, D., Perrissoud, D., Locatelli, V., Torsello, A., Galleyrand, J.C., Fehrentz, J.A. and Martinez J. 2008. New trisubstituted 1,2,4-triazole derivatives as potent ghrelin receptor antagonists. 3. synthesis and pharmacological in vitro and in vivo evaluations. J. Med. Chem. 51, 689-693.

22. Witkowski, J.T., Robins, R.K., Sidwell, R.W. and Simon, L.N. 1972. Design, synthesis, and broad spectrum antiviral activity of 1-.beta.-D-ribofuranosyl-1,2,4-triazole-3carboxamide and related nucleosides. J. Med. Chem. 15, 1150-1154.

23. Cruickshank, R., Duguid, J.P., Marmion, B.P. and Swain, R.H.A. 1975. Medicinal Microbiology, $12^{\text {th }}$ Eds., 2, Churchill Livingstone, London, pp. 196-202.

24. Microbial Type Culture Collection and Gene Bank (MTCC), 2000. Institute of Microbial Technology (IMTECH), Chandigarh, $5^{\text {th }}$ Ed., Medium No-137, pp. 130-138. 